\title{
ADVANCED DIAGNOSTIC SYSTEM FOR UNDERGROUND COMMUNICATION NETWORKS WITH VENTILATION ON DEMAND
}

\author{
By \\ Mohamed Daoud \\ Bachelor of Applied Science \\ Electronics and Electrical Communications Engineering \\ Cairo University, Cairo, Egypt, 2006 \\ A thesis \\ presented to Ryerson University \\ in partial fulfillment of the \\ requirements for the degree of \\ Master of Applied Science \\ in the Program of \\ Electrical and Computer Engineering
}

Toronto, Onatrio, Canada, 2011

(C) Mohamed Daoud, 2011 


\section{AUTHOR DECLARATION}

I hereby declare that I am the sole author of this thesis.

I authorize Ryerson University to lend this thesis to other institutions or individuals for the purpose of scholarly research.

Signature

Mohamed Daoud

I further authorize Ryerson University to reproduce this thesis by photocopying or by other means, in total or in part, at the request of other institutions or individuals for the purpose of scholarly research.

Signature

Mohamed Daoud 


\section{ACKNOWLEDGEMENTS}

I feel deeply indebted to my supervisor, Professor Dr. Xavier Fernando, for his patience, guidance, constant supervision, creative advising and personal involvement throughout the progression of this work. He has been, for me, not only a source of inspiration and encouragement but also a model of ideal academic relations. Much of what I have learned during the progress of this thesis I owe, in fact, to Professor Fernando. Working under the supervision of a very great investigator of the highest academic and human caliber like Professor Fernando has formed me and will continue to be an unforgettable and a unique experience of my life.

I would like to express my great appreciation and gratitude to Mr. Wisam Farjow for his guidance, advice and moral support. Wisam's endless knowledge in mining industry has helped make this work possible. For me he has been not only a mentor but a caring brother as well.

My thanks go to all my Professors at Ryerson University who taught me during my graduate study and to the Department of Electrical and Computer Engineering, Ryerson University for offering all facilities, financial resources and the academic environment.

I would like to express my deepest appreciation to my parents and brothers for their moral support, patience and unconditional love. 


\section{DEDICATION}

To Nancy for giving me the love and care

To Mahmoud for giving me the advice and support 


\title{
ABSTRACT
}

\author{
ADVANCED DIAGNOSTIC SYSTEM FOR UNDERGROUND \\ COMMUNICATION NETWORKS WITH VENTILATION ON DEMAND \\ Mohamed Daoud, 2011 \\ Masters of Applied Science (MASc) \\ Electrical and Computer Engineering Department \\ Ryerson University
}

In this thesis, a novel diagnostic system has been developed to increase the reliability of leaky feeder communication systems in underground mines. The new system is based on three main parts: 1) Diagnostic Receiver Unit (DRU) located in the control room above ground; 2) several Diagnostic Transponder Units (DTU) connected to amplifiers, Power Supply Units (PSU), fans, and sensors; and 3) Communication protocol that was designed for this specific system. The amplifiers, PSU, fans, and sensors in the system cascade will be able to communicate freely with the control room through the leaky cable to send information about their current conditions, and receive configuration messages. A new concept is also presented that provides energy saving in mines; Ventilation-on-Demand where the fans will operate according to the current situation of each section in the mine. This is made possible through a series of interrupt messages that is sent from the DTU attached to the fan/sensor to the DRU in the control room. 


\section{Table of Contents}

Table of Contents vii

List of Tables $\quad$ ix

List of Figures $\quad x$

Acronyms xii

1 The Underground Communication Network 1

1.1 Intoduction . . . . . . . . . . . . . . . . . . 1

1.2 Current System Challenges . . . . . . . . . . . . . . . . . 3

1.3 Contribution of the Thesis . . . . . . . . . . . 5

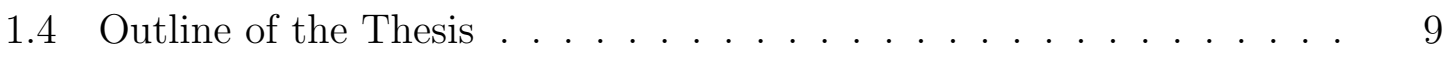

2 Leaky Feeder Systems $\quad 11$

2.1 Introduction to Leaky Feeders . . . . . . . . . . . . . . . . 11

2.2 Current Leaky Feeder System . . . . . . . . . . . . . . . . 15

2.3 Reliability Problem and Other Challenges . . . . . . . . . 18

2.4 Available Solutions for Current Leaky Feeder Networks . . . . . . . . 21

3 Diagnostic System Solution $\quad 25$

3.1 Introduction to Diagnostic System . . . . . . . . . . . . . 25

3.2 Hardware Architecture . . . . . . . . . . . . . . . . . 27

3.2.1 Diagnostic Receiver Unit (DRU) . . . . . . . . . . . . 27

3.2 .2 Diagnostic Transponder Unit (DTU) $\ldots \ldots \ldots \ldots$

3.2 .3 Diagnostic Software Package (DSP) _ . . . . . . . . . . . 32

3.3 Communication Protocol . . . . . . . . . . . . . . . . . . . 34

3.3 .1 Self Configuration . . . . . . . . . . . . . . . . . 37

$3.3 .2 \quad$ Normal Mode . . . . . . . . . . . . . . . . . . . . . . . . 50 
3.3.3 Interrupt Mode . . . . . . . . . . . . . . . . . 59

4 Ventilation-on-Demand $\quad 62$

4.1 Introduction . . . . . . . . . . . . . . . . . . 62

4.2 Ventilation on Demand Using Diagnostic System . . . . . . . . . . . . 63

4.3 Interrupt . . . . . . . . . . . . . . . . . . . 64

5 Performance Evaluation $\quad \mathbf{7 0}$

5.1 Introduction . . . . . . . . . . . . . . . . . 70

5.2 Wake up Time. . . . . . . . . . . . . . . 72

5.3 End-to-End Delay and Throughput . . . . . . . . . . . . . 78

5.4 Component Tolerance Error (CTE) . . . . . . . . . . . . . 80

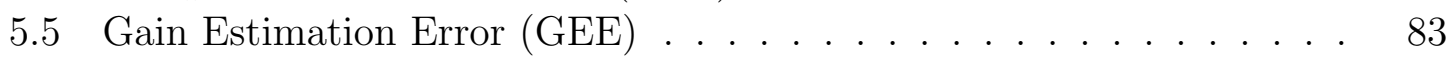

6 Conclusion and Future Work $\quad 85$

6.1 Conclusion . . . . . . . . . . . . . . . . . . 85

6.2 Future Work . . . . . . . . . . . . . . . 87

$\begin{array}{lr}\text { Bibliography } & 90\end{array}$

$\begin{array}{lr}\text { Appendices } & 94\end{array}$ 


\section{List of Tables}

2.1 Leaky Feeder System Frequency Plan . . . . . . . . . . . . . . 17

3.1 DRU Specifications . . . . . . . . . . . . . . . . . . . . . . . . 29

3.2 DTU Specifications . . . . . . . . . . . . . . . . . . . 32

3.3 Communication Protocol Specifications . . . . . . . . . . 35

3.4 Bits 2-4 of the Control Field . . . . . . . . . . . . . . . 36

3.5 Data Configured During the Write Cycle . . . . . . . . . . . 53

3.6 Data Read During Read Cycle . . . . . . . . . . . . . . 58

4.1 Data Read During Interrupt Mode-Read Message . . . . . . . . 67

4.2 Data Configured During the Write Cycle-Interrupt Mode . . . . . . 69

5.1 System Configuration . . . . . . . . . . . . . . . . . 71

5.2 Measured Values vs. Simulation Results part $1 / 2 \ldots \ldots$. . . . 73

5.3 Measured Values vs. Simulation Results part 2/2 . . . . . . . . 74 


\section{List of Figures}

1.1 Leaky Feeder Network with Diagnostic System Installed _ . . . . . 8

2.1 Leaky Cable Structure . . . . . . . . . . . . . . . . . . . . . 12

2.2 Basic Operation of Leaky Feeder System . . . . . . . . . . . . 14

2.3 Typical System Arrangement _. . . . . . . . . . . . . . . 15

2.4 General Layout of Leaky Feeder Underground Network Providing Various Services . . . . . . . . . . . . . . . . . . . 16

2.5 System Bands Orientation Layout . . . . . . . . . . . . . . 17

2.6 Multiple Upstream and Downstream Reference Pilot System with AGC 19

3.1 DTU Connected to BDA in Leaky Feeder Network . . . . . . . . 27

3.2 DRU Architecture . . . . . . . . . . . . . . . . . . . . . 28

3.3 DTU Architecture . . . . . . . . . . . . . . . . . . . . 31

3.4 General Message Format . . . . . . . . . . . . . . . . 35

3.5 Self Configuration Pilot Signal at DRU and the Attenuated Version at

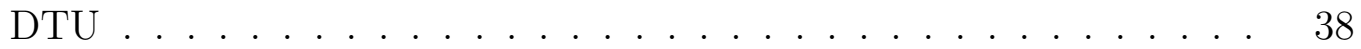

$3.6 \quad$ RF killer in place on the downstream . . . . . . . . . . . . . 39

3.7 Leaky Cable Longitudinal Loss Chart . . . . . . . . . . . . . . . 41

3.8 Self Configuration Message Sequence . . . . . . . . . . . . . . 42

3.9 SET_D Message Format . . . . . . . . . . . . . . . . . . . 43

3.10 RRES Message Format . . . . . . . . . . . . . . . . 44

3.11 ACK_SET_D Message Format . . . . . . . . . . . . . . 45

3.12 Self Configuration Operation Mode . . . . . . . . . . . . . 47 
3.13 DRU Flowchart . . . . . . . . . . . . . . . . . . . . 48

3.14 DTU Flowchart . . . . . . . . . . . . . . . . . . . . . . 49

3.15 Normal Mode- Write Cycle Message Sequence . . . . . . . . . . . 51

3.16 Format of WRITE Message . . . . . . . . . . . . . . 52

3.17 Format of ACK Message . . . . . . . . . . . . . . . . 53

3.18 Normal Mode- Read Cycle Message Sequence . . . . . . . . . . 55

3.19 Format of REQ Message . . . . . . . . . . . . . . 55

3.20 Format of DATA_READ Message . . . . . . . . . . . 57

3.21 Write Mode Sequence . . . . . . . . . . . . . . . . . . 60

3.22 Read Mode Sequence . . . . . . . . . . . . . . . . . 60

4.1 Interrupt Message Sequence . . . . . . . . . . . . . . . 65

4.2 Interrupt Message Format . . . . . . . . . . . . . . . 66

4.3 Interrupt Mode Sequence . . . . . . . . . . . . . . . . . . 69

5.1 Leaky Feeder System Setup _ . . . . . . . . . . . . . . . . . 72

5.2 Percentage of DTU Wake up Time to Total Time for Different System Sizes- Self Configuration Mode . . . . . . . . . . . . . . 75

5.3 Percentage of DTU Wake up Time to Total Time for Different System Sizes- Normal Mode- Write Cycle . . . . . . . . . . . . . . . 76

5.4 Percentage of DTU Wake up Time to Total Time for Different System Sizes- Normal Mode- Read Cycle . . . . . . . . . . . . . . . 77

5.5 Extra Processing Time for Different System Sizes- Interrupt Mode . . 78

5.6 Average End-to-End Delay in the Network Over One Hour . . . . . . 79

5.7 Average Throughput in the Network over an Hour . . . . . . . 80

5.8 CTE Simulation Analysis . . . . . . . . . . . . . . . . . 82

5.9 GEE Simulation Analysis _ . . . . . . . . . . . . . . . 84 


\section{Acronyms}

MINER Mine Improvement and New Emergency Response

WSN Wireless Sensor Networks

TTE Through The Earth

ELF Extremely Low Frequency

PSU Power Supply Unit

CMHS Coal Mine Health and Safety

DRU Diagnostic Receiver Unit

DTU Diagnostic Transponder Unit

MSHA Mine Safety and Health Administration

PTT Push To Talk

DOCSIS Data Over Cable Service Interface Specification

CMTS Cable Modem Termination System

BDA Bi-Directional Amplifier

RF Radio Frequency

AGC Automatic Gain Control

ASC Automatic Slope Control

LED Light Emitting Diode

DSP Diagnostic Software Program

GUI Graphical User Interface

MTW Maximum Time to Wait

CRC Cyclic Redundancy Check

ACK Acknowledge 


\begin{abstract}
INT Interrupt
VNA Vector Network Analyzer

CTE Components Tolerance Error

GEE Gain Estimation Error
\end{abstract}




\section{Chapter 1}

\section{The Underground Communication Network}

\subsection{Intoduction}

After a series of recent world wide mine tragedies, ranging from collapsing mines in China and Colombia to trapped miners in Chile, the importance of robust and reliable underground communication networks has become evident once again. A reliable underground communication network in mines will not only facilitate the day to day operations, but will also help save many lives. However; designing reliable and robust underground network has always been a challenge due to the special nature of mines.

Mining industry is not only important for job creation but is also essential to sustain humanity as well, by supplying minerals that the world of today could not exist without. Mining industry contributes to the economies of different countries and help build new communities. Mining is extremely important for Canadian economy. Canada is considered a major player in the mining industry worldwide. Canada produces about 60 minerals. It ranks first among all mineral producing countries 
in the number of minerals produced. In addition, Canada is the largest exporter of minerals, with more than $20 \%$ of production shipped to world markets [9].

In a typical year, the mining industry is responsible for almost $20 \%$ of Canada's total export earnings. As for the employment rate, over $70 \%$ of the mines are owned by Canadians and approximately 108,000 Canadians are directly employed in the mining industry [9].

Products from mining industry power the automobiles and heat buildings, and supply the manufacturing sector; the high tech industries and even the better known resource industries are all dependent, in some way, on the mining industry. The mining industry will continue to be an important support to the economy. Mining is taking full advantage of the quick expansion of computers and microelectronics. These technologies are found in nearly every aspect of mineral development activity - from exploration methods, through production, mineral processing and even marketing. Computers and related equipment now have a lot of different applications in geophysical logging, geochemistry, geological mapping, surface contouring, and most important communications.

Communication in mines is the intersection point for all of the aforementioned concerns and considerations, since it is used at every stage of mining operations. Day-to-day operations, extracting and moving the product are handled with the aid of communications, which increases the productivity. Remote monitoring and control operations depend completely on communications, too [21, 22]. 


\subsection{Current System Challenges}

Following tragic events in many underground mines such as those at the Darby, Alma, Sago coal mines, and recently the big incident of Chile, the need for reliable communications systems in underground mines became evident. Such previous incidents and many more triggered the Mine Improvement and New Emergency Response (MINER) Act of 2006 in the United States. The most important aspect of the MINER Act is providing reliable communication for miners before and after tragic incidents. Reliable communication has always been a challenge in underground mines due to changing topologies and the harsh environment. In addition, disasters disable electricity and communications cables, block tunnels and cause fire. These may damage the communication infrastructure which hampers rescue efforts and endangering lives. Therefore, durable wireless solutions have been investigated and researched by both the industry and the academic institutions to provide reliable communications in underground mines.

There are various communication systems for underground mines ranging from wired systems like paging phones to wireless systems like Wireless Sensor Networks (WSN) and also hybrid systems like leaky feeders. Currently, three main systems are

widely used: leaky feeders, Through The Earth (TTE), and WSN. While TTE is used in case of disaster recovery to locate trapped miners and wireless sensors networks are used for sensing the mine conditions, the leaky feeder system can be considered 
the most popular communication system used for day-to-day operations. Leaky feeders have long successful history in mine communications [11]. Due to the ability of leaky feeder networks to carry voice, video, and data in underground networks, it has emerged to be the most popular communication system in mines since the early 90 's. However; leaky feeder system is not reliable and its problems are hard to diagnose.

Starting with the TTE system; it is used to employ an effective and timely rescue operation when miners are trapped underground after a disaster. The rescue team above ground use Extremely Low Frequency (ELF) waves to penetrate earth and locate the trapped miners and also to establish communications with them. It may be the best option for rescue operation, however; it requires huge transmitter power and antenna size. Another disadvantage is the inaccuracy since the TTE depends on the depth, frequency, and the characteristics of the ground. For WSN, it consists of several repeater nodes which are able to store and forward information to other nodes. WSN requires limited human intervention for system configuration and is both self-healing and robust. Providing low data rate is a disadvantage that limits it to specific applications. WSN shows different performance depending on the mine gallery geometry for instance it shows poor performance around the corners and at pillar areas. Finally, when it comes to leaky feeders different companies have developed different systems, but they all have the same basic idea; coaxial cable runs all the way from a control room above the ground through different parts of the mine. This cable radiates over its entire length allowing signals to be leaked out and into the cable providing two-way communications and acting as both antenna and transmission medium at the same time. Signal attenuates while traveling through 
the cable which requires bi-directional amplifiers every $350 \sim 400$ meters. These amplifiers are powered by DC voltage supplied through the cable's center conductor [14], which in most existing leaky feeder systems operates at 30-300 MHz. In addition to amplifiers the leaky feeder cable is connected to Power Supply Units (PSU) as well.

Reliability, and robustness are two essential characteristics of underground communications networks, however; they have not yet been fully explored. There are two main reasons for that. The first is due to the complexity of communication networks in mines as the environment is extremely harsh and the geometry of the mine gallery keeps changing. The second reason is purely historical: which was the deregulation of the industry; it was only major accidents that triggered the federal government to introduce different acts. For example the famous mine accidents in the 1960's leading to the Coal Mine Health and Safety (CMHS) Act of 1969 [15], and the Mine Improvement and New Emergency Response (MINER) Act of 2006.

\subsection{Contribution of the Thesis}

The advanced monitoring system presented here is an integrated novel solution for monitoring underground communication networks. This is based on leaky feeder system and is designed to utilize the leaky feeder's infrastructure to monitor itself while providing ventilation on demand. This system solves the reliability problem in leaky feeders by providing accurate real time information about the leaky feeder network underground to the operator in the control room above ground. More over, this will assist them in taking decisions and troubleshooting the network problems. 
Intelligence is introduced to the system by adding a Diagnostic Receiver Unit (DRU) connected to the leaky feeder headend located in the control room above ground and several Diagnostic Transponder Units (DTU) connected to every amplifier, PSU, fan, and sensor in the system. The DTU is able to acquire specific information from the node connected to it and sends it real time to the DRU. Using a software-friendly interface the DRU will present real time data to the operator in the control room allowing better trouble shooting and assistance in the decision making process. Losses along the leaky feeder cable will be detected using a novel technique.

This Diagnostic System operates in three modes: self configuration mode, normal mode, and interrupt mode. Self configuration mode detects network layout, number and type of nodes, distance between nodes, and losses. Normal mode allows DRU to read information from all nodes and change any parameters remotely from the control room. Interrupt mode enables any DTU to interrupt the DRU and send to it critical information about the node connected to it. This diagnostic system will also provide ventilation on demand as it will switch the fans on or off according to the information it receives from sensors through the DTU. It is up to the authors' knowledge that such a complete system never been investigated before. Figure 1.1, shows an overview of the system.

Ventilation on Demand concept is to control the amount of ventilation and power to reduce the energy requirements as mining depth increases. Our protocol is based on the ability to control the volume of air and thus power requirements of the specific activities required for the mining process. To accomplish this the fan needs to be 
informed of the current level of gases, temperature, and other air quality parameters in each section of the mine. This is accomplished using various temperature and air quality sensors.

Below is a summary of the main thesis contribution that will be presented in details in the following chapters:

- Hardware design of DRU

- Hardware design of DTU

- Smart communication protocol with different modes

- Normal mode: enables polling all DTU's and reading their data in addition to configuring them remotely

- Self configuration mode: detects the layout of the underground network, calculates losses, distance between nodes, number of splitters, and does accurate trouble shooting

- Interrupt mode: for important alarms

- Ventilation on Demand is a feature built on the interrupt mode that will provide aggressive savings by cutting the power consumed in mines and helps in introducing the concept of "Green Mines." 


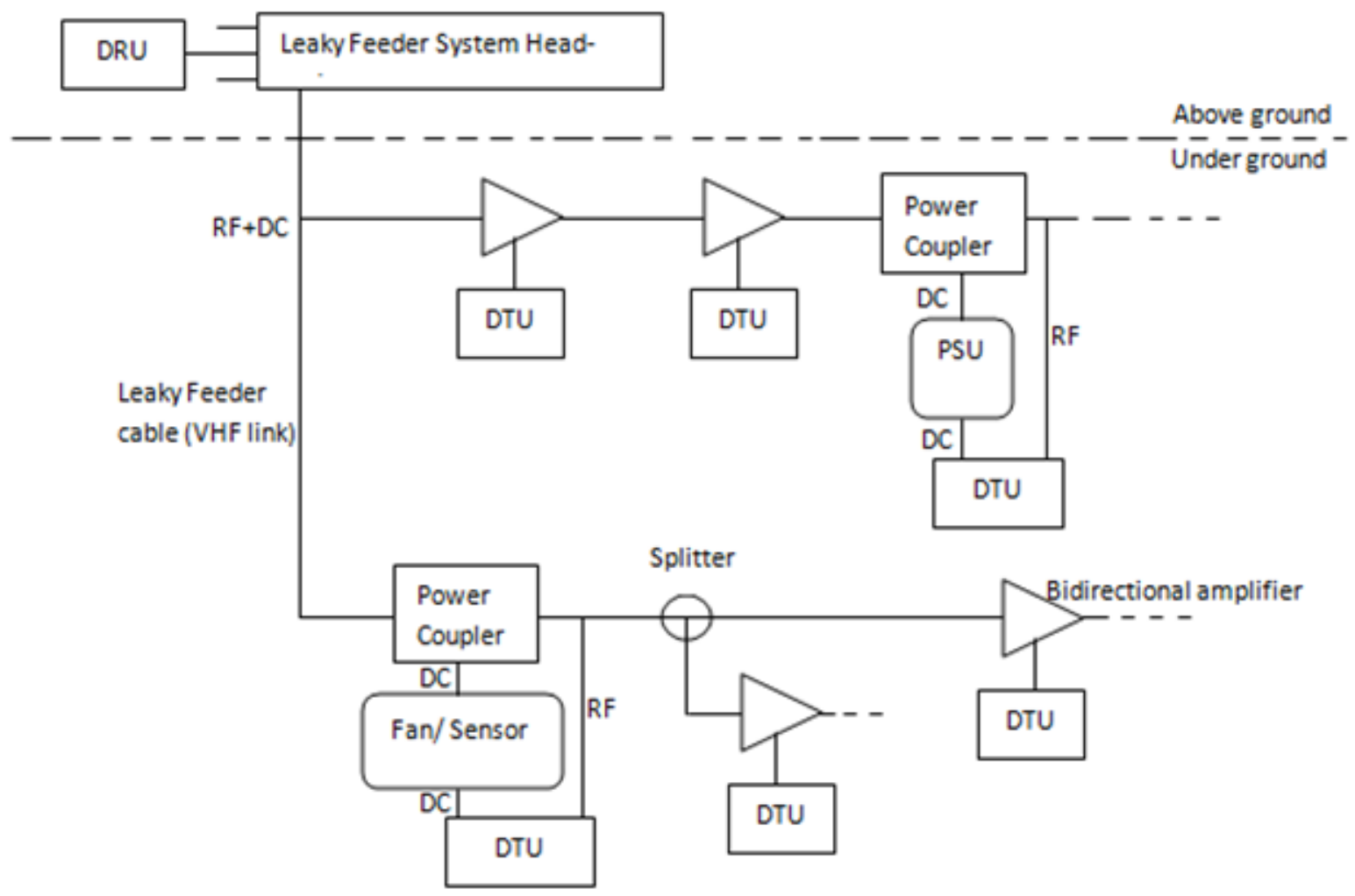

Figure 1.1: Leaky Feeder Network with Diagnostic System Installed 


\subsection{Outline of the Thesis}

\section{Chapter 1: The Underground Communication Networks}

This chapter gives a general overview of the mining industry in Canada and communication networks currently deployed in the confined spaces of underground mines. The challenges encountered with nowadays underground communication networks is briefly described.

\section{Chapter 2: Leaky Feeder System}

This chapter gives an introduction to leaky feeder theory. Also, current network configuration and the associated problems are discussed here.

\section{Chapter 3: Diagnostic System Solution}

In this chapter a new and novel solution is presented to monitor, control, and add reliability to underground leaky feeder networks. The diagnostic system is described here in terms of: hardware architecture of different nodes and the smart communication protocol along with its different modes.

\section{Chapter 4: Performance Evaluation}

Discussion of the diagnostic system performance is presented here. Evaluation of the communication protocol under different types of errors is also shown in this chapter.

\section{Chapter 5: Ventilation on Demand}

This chapter introduces the concept of ventilation on demand and explains its effect on mines. Our design of ventilation on demand mode is presented here. We show how 
ventilation on demand can be built on top of the interrupt mode that we designed as part of our communication protocol.

\section{Chapter 6: Conclusion and Future Work}

This chapter summarizes the results and conclusions of the thesis work. Some suggested research activities are presented to carry on further work. 


\section{Chapter 2}

\section{Leaky Feeder Systems}

\subsection{Introduction to Leaky Feeders}

Due to the fact that wireless and mobile communications in confined space environments, such as underground mines and tunnels, tend to have different philosophy to that of the open space wireless communications, leaky feeder became the most used communication solution in mines. The leaky feeder system has a long history of success in tunnel applications [11], and it has been used in some coal mines for routine communications since the 1980s [14].

VHF and UHF signals propagate very poorly in mines; thus, signal transmission is confined to mine openings like entries and crosscuts. Therefore, a leaky feeder cable must be strung throughout a mine wherever access to communications is required. Leaky feeder cable also known as "Radiating Cable," functions as both antenna and transmission line. RF energy is simultaneously transmitted down the radiating cable and radiated from all points into the surrounding space. Slots cut into the outer

conductor of the coaxial cable allow controlled levels of electromagnetic energy to be 
radiated both out of and into the cable.

The amount of radiation is quantified by the coupling loss. The coupling loss is defined as the difference between the power transmitted into the cable and the power received by a $\frac{\lambda}{2}$ dipole antenna located at a distance 2 meters from the cable [7]. The structure of a typical leaky cable is that the inner conductor is covered by a layer of insulation foam. This is then covered by a layer of outer conductor, and finally insulated by a cable jacket. A typical leaky cable structure is shown in Figure 2.1.

\section{1- Cable Support 2- Outer Conductor 3- Insulation Foam 4- Inner Conductor 5- Radiating Slots 6- Cable Jacket}

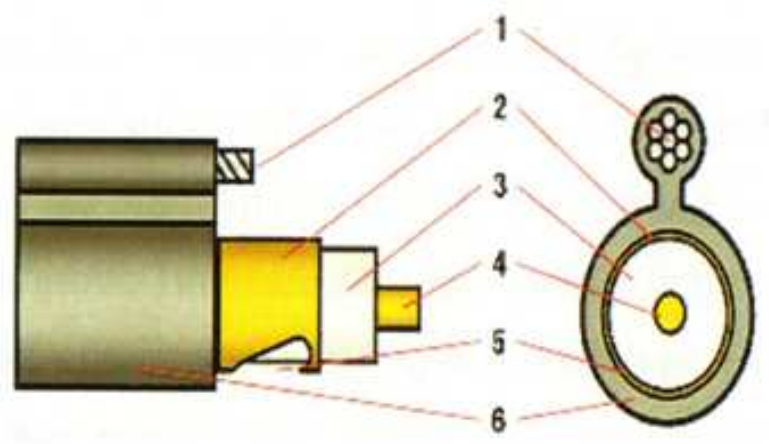

Figure 2.1: Leaky Cable Structure

The leaky feeder network is a combination of leaky feeder cable and amplifiers. Other components are added as well, like PSU, however; the backbone of the system compromises leaky feeder cable and amplifiers only. The inherent loss of signal must be compensated for and this is achieved by the insertion of line amplifiers. The amplifiers are designed to compensate for a certain length of cable. Correct installation of the system will ensure that a consistent signal level is maintained throughout the network, hence throughout the mine [4]. 
Different loss models can be used for mines, but the propagation loss model, the most common model, is considered here. It will be easily noticed that the degree of attenuation throughout the leaky cable is lower than that of free-space propagation in the mine. This in turns means that the leaky feeder system increases the propagation range $[22,18,5]$.

The basic operation of leaky feeder networks is as shown in Figure 2.2, where the headend of the feeder cable connects to the base station located in the control room on the surface. The connection between the base station and the mine's leaky feeder at the mine entry is usually a non-radiating coaxial cable [14]. The base station is responsible to coordinate the flow of communication within the mine in terms of assigning channels, paging, providing quality of service, and connecting circuits. Again, in Figure 2.2, an example is provided where miner 1 and miner 2 are at two separate physical locations while still talking to each other through the leaky feeder network. It is a duplex communication mode where the call is carried on an uplink frequency to the base station which then forwards it through a different downlink frequency to the specified radio device.

When designing a leaky feeder communication network underground, there are many parameters the designer has to pay attention to like the number of amplifiers, PSU, distance between amplifiers, partitioning of cells, etc. As the shape of the leaky feeder network is somehow complex and have few different arrangements. The general arrangement of the leaky feeder network is that the system is sectioned off into cells that are separated by barriers. A barrier is a device that allows radio signals to run 


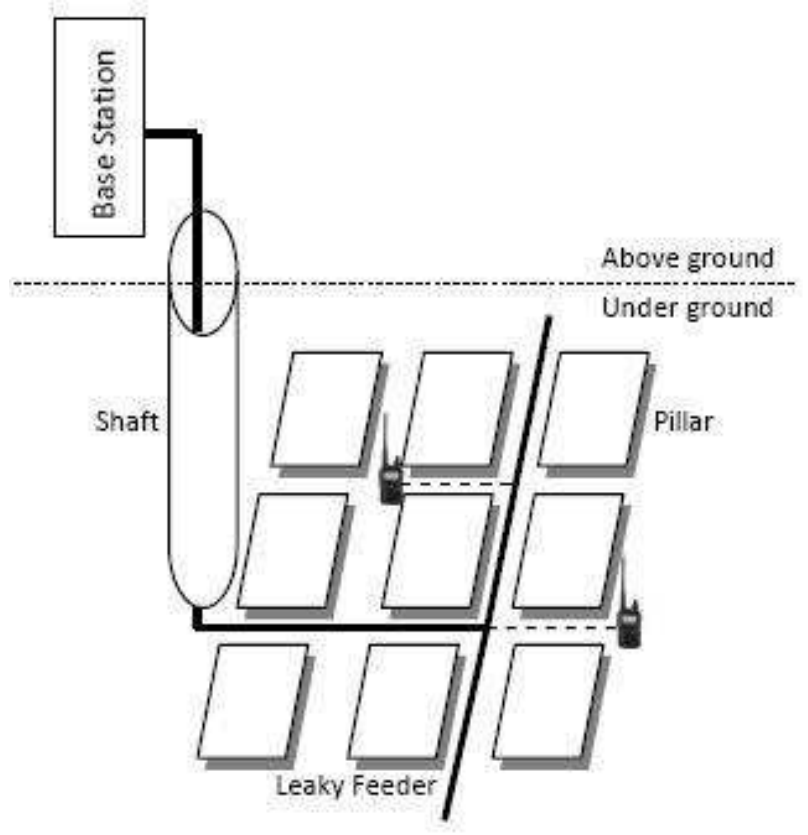

Figure 2.2: Basic Operation of Leaky Feeder System

through the feeder cable but blocks DC power from transferring between cells [13]. Thus, all amplifiers in a given cell are powered from a single power supply dedicated to that cell. There are some injection points that allow DC voltage from the power supply to be imposed on the center conductor of the leaky feeder cable. Figure 2.3, shows a typical leaky feeder arrangement.

Since the system must operate after a catastrophic event, each power supply requires a battery backup. Lead-acid batteries are used for this application. The batteries and their associated charging equipment are housed in an Mine Safety and Health Administration (MSHA) approved, explosion-proof enclosure within each cell location. All other communications components are approved as intrinsically safe. A typical spacing between adjacent power supplies is 1500 meters. 


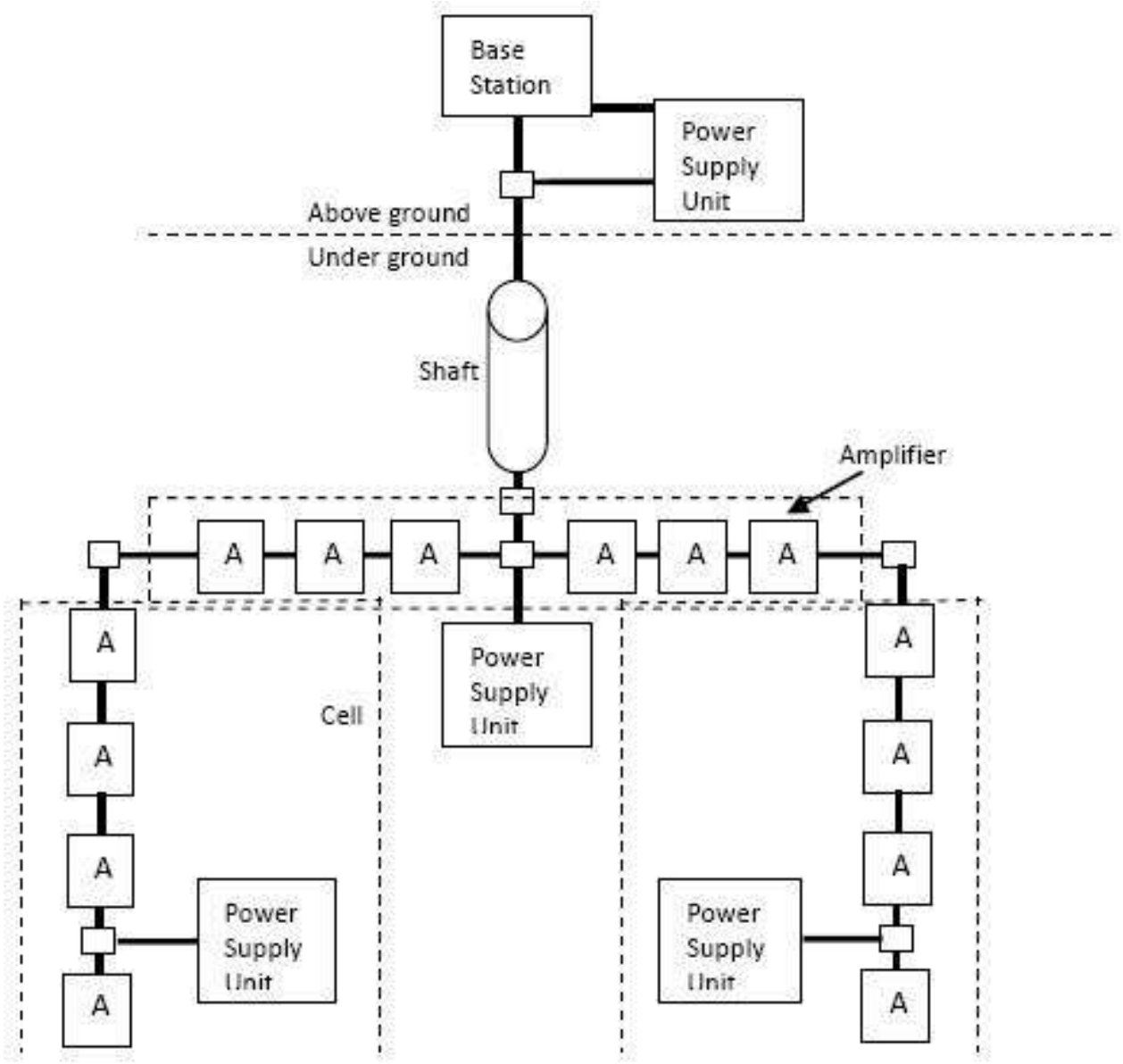

Figure 2.3: Typical System Arrangement

\subsection{Current Leaky Feeder System}

Nowadays, leaky feeder system can do more than just the basic operation; it can transfer data, video, PTT (Push To Talk), high speed internet, underground video conferencing, and much more. The high speed data communication is made possible by utilizing Data Over Cable Service Interface Specification (DOCSIS) standards to 
deliver Ethernet connectivity based on Cable Modem Termination System (CMTS) in leaky feeder systems. Typically, there are four bands of amplifications in the system amplifiers to compensate for the system losses. In the CMTS system, there are two bands dedicated to provide full-duplex voice communications (upstream and downstream) and two bands to provide the CMTS high speed data communications in the upstream and downstream directions. Figure 2.4, shows general layout of leaky feeder underground network providing various services.

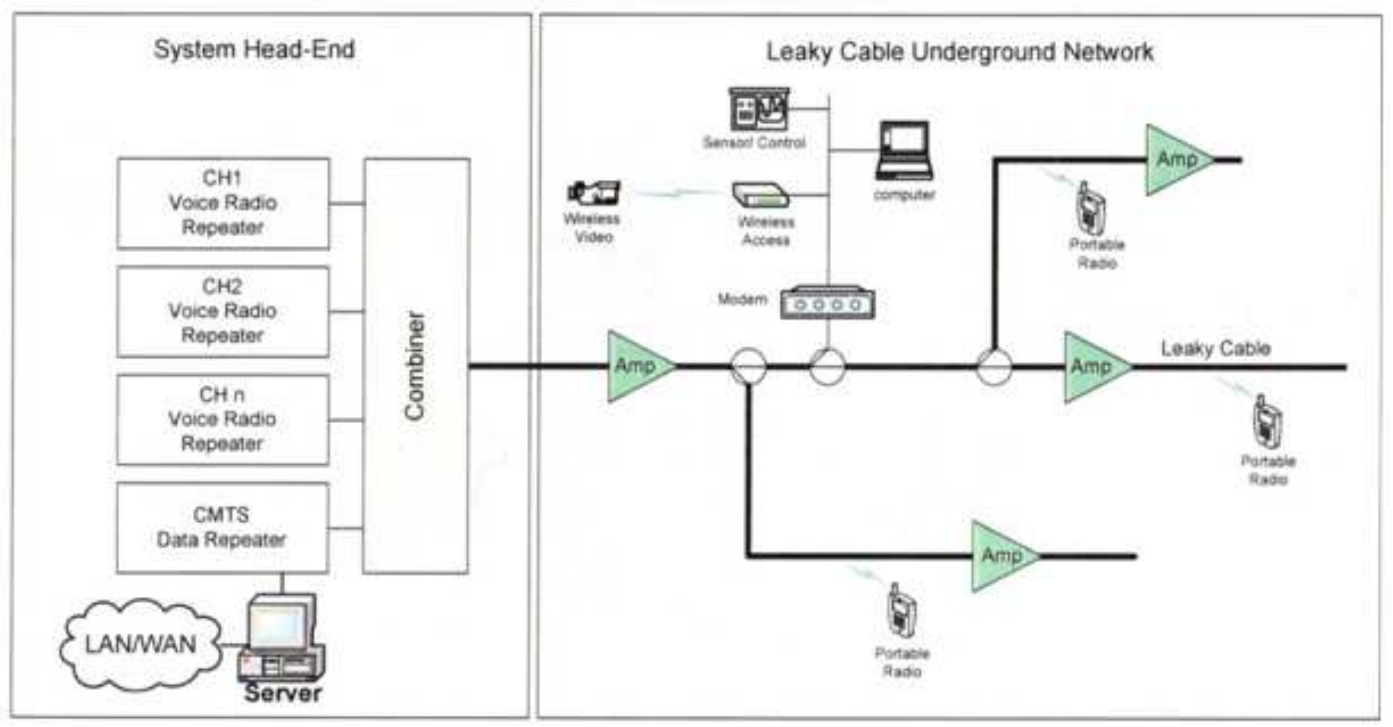

Figure 2.4: General Layout of Leaky Feeder Underground Network Providing Various Services

The four distinct RF bands in the system are required to provide RF paths for voice and data communications. Table 2.1, shows the system frequency plan, it lists the frequency range for each band. While the upstream and downstream orientation layout of these bands is shown in Figure 2.5.

The current systems achieve coverage by utilizing in-line Bi-Directional Amplifiers 
Table 2.1: Leaky Feeder System Frequency Plan

\begin{tabular}{|l|l|l|}
\hline Band No. & Band Name & Frequency \\
\hline 1 & Upstream Data & $5-42 \mathrm{MHz}$ \\
2 & Downstream Voice & $155-158 \mathrm{MHz}$ \\
3 & Upstream Voice & $172-175 \mathrm{MHz}$ \\
4 & Downstream Data & $220-232 \mathrm{MHz}$ \\
\hline
\end{tabular}

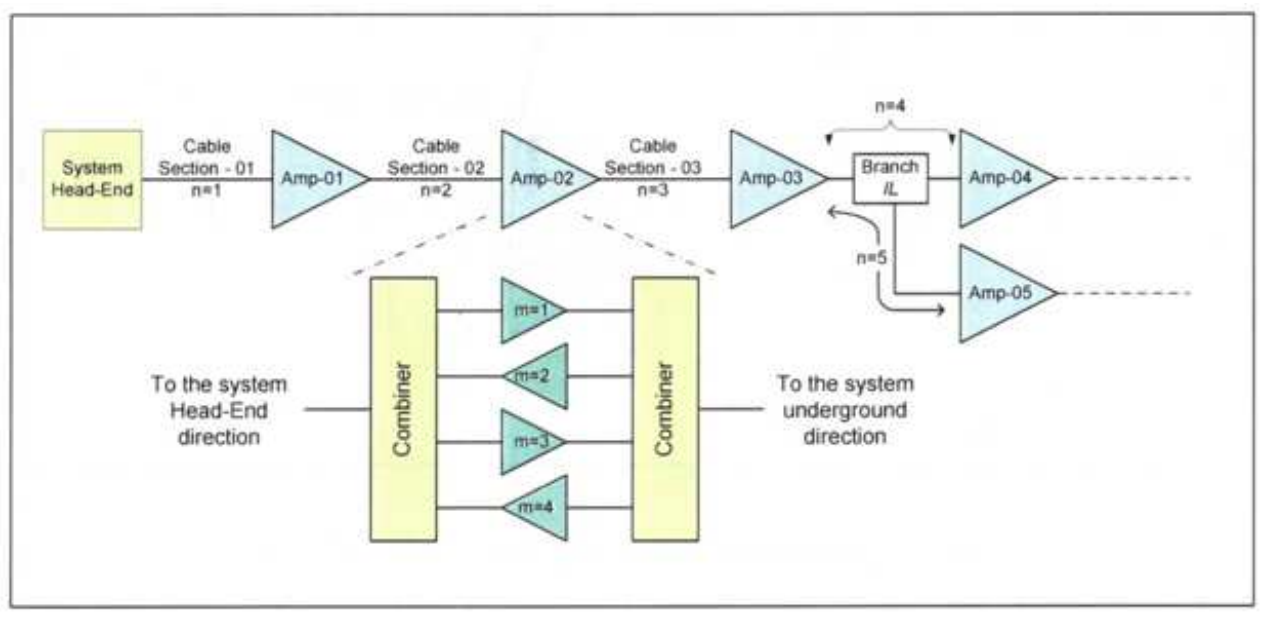

Figure 2.5: System Bands Orientation Layout

(BDA) distributed along the underground tunnels and galleries in a multi- cascaded topology. RF signals are amplified in both directions (upstream and downstream) to maintain adequate signal levels to compensate for fixed or dynamic system losses.

Maintaining consistent RF levels in any system require methods of controlling the gain and slopes of the amplifiers. These requirements led to the design of special leaky feeder communication network circuits, where the primary ideal function is to maintain a constant signal level at the output, regardless of the signal's variations at the input of the system. These circuits are known as Automatic Gain Control (AGC) and Automatic Slope Control (ASC), AGC/ASC circuits and are currently used in all leaky feeder communication systems. 


\subsection{Reliability Problem and Other Challenges}

In leaky feeder cables the quality of radio communications vary with the level of the signal travelling through the cable. Temperature variation can manipulate the signal level by changing the longitudinal loss characteristics of the cable. Also external noise caused by mine machineries can vary the signal level. Mines are very dynamic in the way they grow; there are daily expansions which require more cables and branches to be added to the system. This requires more amplifiers to compensate the losses where AGC is used in the system. Many AGC and ASC circuits where introduced in the leaky feeder systems to compensate for and overcome the variations in the levels of the RF signals. The most common approach in deploying AGC/ASC is by utilizing reference RF carriers normally referred as Pilots [16]. These reference pilots with known value propagate through the entire system and are used by each amplifier as measuring references to correct the gain and slopes of the amplifier's pass bands.

Challenges exist in deploying AGC/ASC functions in the current leaky cable systems. From system level point of view, the reliance on the reference pilots within the AGC/ASC circuits creates major problems, especially for multi-branched large leaky cable systems, which is the case in most underground mines topology. In these types of systems, it is required to install multiple reference pilot generators at the system headend and at the end of each branch in the system. In case of upstream direction, the problem occurs at the point of the system branching where the pilot carriers from each branch superimpose. The accuracy of the reference pilots is affected. This affects 


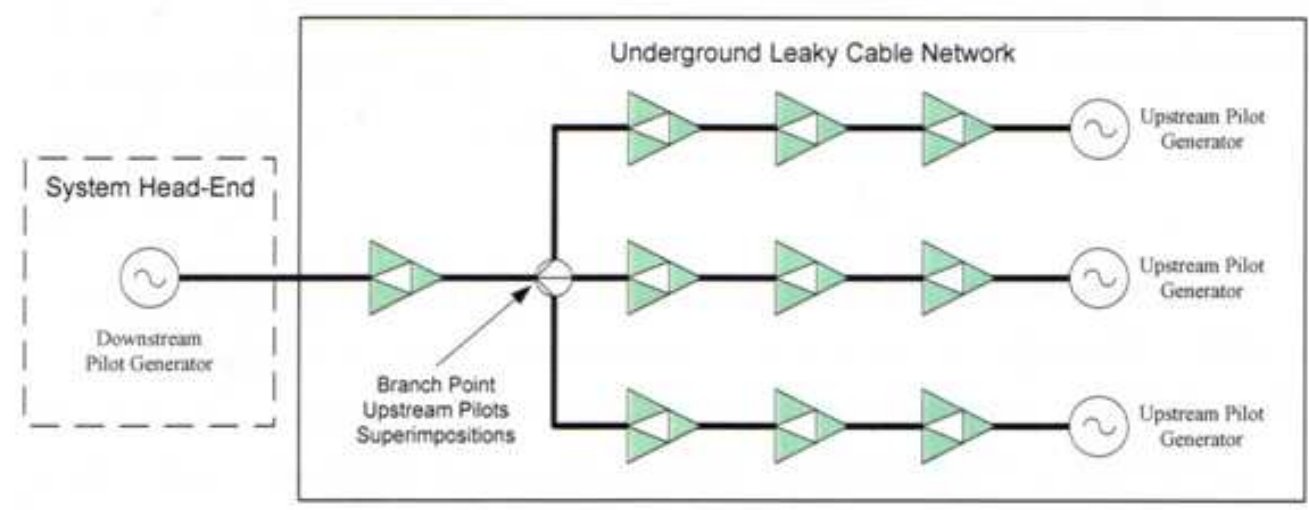

Figure 2.6: Multiple Upstream and Downstream Reference Pilot System with AGC

the operations of the AGC circuits in the subsequent amplifiers and result in instable system operations [7]. Figure 2.6, shows an AGC multiple reference pilot system.

The other major problem in the current system is controlling these amplifiers and changing their parameters remotely. Currently, these amplifiers are controlled locally. Technician has to go underground to troubleshoot the problems with the upstream pilot generator and change its parameters. The rate of sending the pilots has to be changed by visiting underground as well. It often happens that collision occurs between pilots generated at different upstream pilot generators leaving the operator in the control room with absolutely no information about the status of the amplifiers. Therefore; the operator would not be able to set their gain from the control room.

The operator in the control room above ground will have no information about the status of amplifiers and the network in general unless the pilot is received successfully. Even in this case; the operator will have no control over the amplifiers.

In summary, the current system has been in use since the 90's and therefore has many 
prolonged problems. Almost all troubleshooting has to be done by sending a technician underground to the mine. Also, no real time information about the network is available to the operator in the control room above the ground. Detecting the losses in the network or the number of branches or number of nodes is almost impossible without prior knowledge of the network. Any changes in the mine layout like expansion or shutting down a certain area cannot be detected automatically. The current pilot system is not reliable and sometimes pilots from different upstream generators collide or superimpose at branching points. This leads to errors and wrong information about some amplifiers.

Some efforts have been done to enhance the current system, but still they all are based on the same idea of pilot signals which is the core problem. Surveying some of the previous work done we can see that in [21], Craig A. Winkelmann et al. tackled the reliability problem by implementing a self-testing algorithm for microprocessors in mine monitoring systems; this technique assumed that problems occur at microprocessor level only which is not always the case. In [2], Robert S. Chapman et al. presented a methodology that could be used for measuring the performance of a monitoring system, Again in [1], they continue their efforts to develop an underground mine monitoring and control testing criteria. In [19], J. Sottile et al. surveyed a number of available fault monitoring and diagnosis equipment at that time, where they compared the model-based approach to the neural nets. Both approaches lack reliability and most of the systems address one problem at a time. In [6], T. H. Dubaniewicz et al. introduced a system based on fiber optics to monitor air quality in mines, fiber optics are quite reliable, however; it is extra hardware which means extra cost. 


\subsection{Available Solutions for Current Leaky Feeder Networks}

In this section, few interesting solutions that are already available are described.

Smart Com by Varis Mine Technology Ltd. is a hybrid system that incorporates leaky feeder technology and Ethernet technology. The Ethernet network is used to provide diagnostic capabilities to the amplifiers underground. Information regarding the amplifiers is sent to another node in the control room above ground. However; this system lacks the remote configuration capability as the operator in the control room cannot change parameters of the amplifiers. Only read their information. Another drawback is that this system ignores the PSU and only focuses on amplifiers. Contrary to our diagnostic system, this system does not provide self configuration or support ventilation on demand.

Beckercom by Becker Mining Systems AG is a leaky feeder system that only provides local diagnostic. Operator has to go underground and connect their equipment to diagnose each node. They have a set of LEDs and flash lights on each amplifier that are clearly seen which makes high level diagnosing easy to anyone like regular mine workers. These LEDs show the amplifier performance and can be seen from passing vehicles. This system lacks all remote and troubleshooting capabilities that results in huge maintaining and running cost. 
SIAMnet by Cattron Group International Inc. is another leaky feeder product that optimizes leaky feeder channels for best performance and supports RFID as well. However; it still uses the old pilot system, thus; it provides no diagnostic capability in addition to having no reliable amplifier that cause errors in the system.

Infinity Series is an amplifier developed by Mine Site Technologies Inc., to be used in their leaky feeder system. They sell this as part of their leaky feeder solution as a standalone product as well. It has a built-in diagnosing algorithm that can be accessed locally by sending a technician underground. They claim this amplifier has a longer than usual life time since it is covered by a special dielectric casing that protects the amplifier from errors caused by temperature variation and harsh underground environment.

MR90 by FHF Bergbautechnik GmbH \& Co. KG is a complete leaky feeder system with no diagnostic capability. It has a special technology that allows the spacing between amplifiers to reach up to 1,000 meters compared to the current system that support spacing up to 350 meters. It still depends on the pilot system.

Minecom Leaky Feeder System by Minecom Ltd is the normal classical leaky feeder system that can provide voice and data services but with absolutely no monitoring or diagnosing. This is built on the old pilot system. Therefore; it suffers from the same problems. 
There are some other systems that provide different kinds of diagnosing by using extra hardware, such as optical fiber that will boast the cost. Also, for underground environment monitoring a separate network is needed which means extra cost and more problems.

After we have done a survey for all commercially available solutions in the market we have found that there is no complete solution for monitoring, and controlling the communication network in mines along with providing ventilation on demand. Currently commercially available solutions lack the remote capability to not only read the amplifiers status but also to configure them. Also available solutions focus only on amplifiers and ignore the PSU which is an important part in the underground networks. No available solution provides ventilation on demand; usually it is a separate product which means extra cost to mining companies.

Addressing the above mentioned problems we introduce a novel diagnostic system which gives the operator in the control room full control over all network elements like amplifiers, PSU, fans, and sensors. The system can not only read their information but set their parameters as well. Therefore, no more upstream pilot generators is required since gain control can be done through the control room above ground. No more technicians are needed to go underground for trouble shooting and setting the pilot generators. Ventilation on demand is introduced as part of the integrated solution that will provide huge power savings. All this will happen using the same infrastructure in place right now with minor changes. Therefore, the proposed system uses the infrastructure to monitor itself. 
The idea we introduce here is a revolutionary concept that will change the way underground leaky feeder communication networks operate forever. 


\section{Chapter 3}

\section{Diagnostic System Solution}

In this chapter we present the theory behind the diagnostic system. Details about the hardware and firmware and the communication protocol are presented here. For the communication protocol, which is our core work, we show different modes, cycles, and discuss the packet format of each message.

\subsection{Introduction to Diagnostic System}

Diagnostic System is a monitoring sensor network which collects data from critical nodes of leaky cable network for further processing and optimization of network parameters. This system is capable of monitoring, diagnosing and failure prediction from a remote location such as a control room. The diagnostic system consists of three main components:

1. Diagnostic Transponder Unit (DTU)

2. Diagnostic Receiver Unit (DRU)

3. Diagnostic Software Package (DSP) 
The DTU is installed inside some units (critical nodes) such as the BDA and PSU. The DTU collects data such as RF level, temperature, etc. and pass them to the headend controller i.e. DRU. The DRU stores data in database for further processing by operator through DSP. The DTU can further act as I/O device to control the functionality of BDAs or PSUs remotely by DSP. Detailed diagram of the diagnostic system installed in a leaky feeder underground network can be seen in Figure 1.1.

Our diagnostic system was developed to provide advanced monitoring that enables operator to acquire up-to-date information about amplifiers, PSU, fans, and sensors, while keeping the network simple and maintaining the cost-effective nature of leaky feeders.

The diagnostic system uses a special communication protocol that was also developed here. It provides self configuration capabilities in which the layout of the network is automatically detected with all losses and number of splitters reported. Furthermore, it enables troubleshooting, changing and reading parameters of any nodes remotely, and ventilation on demand.

This has to happen while maintaining the same infrastructure, and only applying minimal modifications. It has to be back-to-back compatible with current leaky feeder networks while still being cost effective. Figure 3.1, shows the connection between DTU and some BDA. For a detailed diagram, see Figure 1.1. 
The Diagnostic System saves energy by providing ventilation on demand. This is huge energy savings since ventilation is responsible for around $60 \%$ of power consumption in mines.

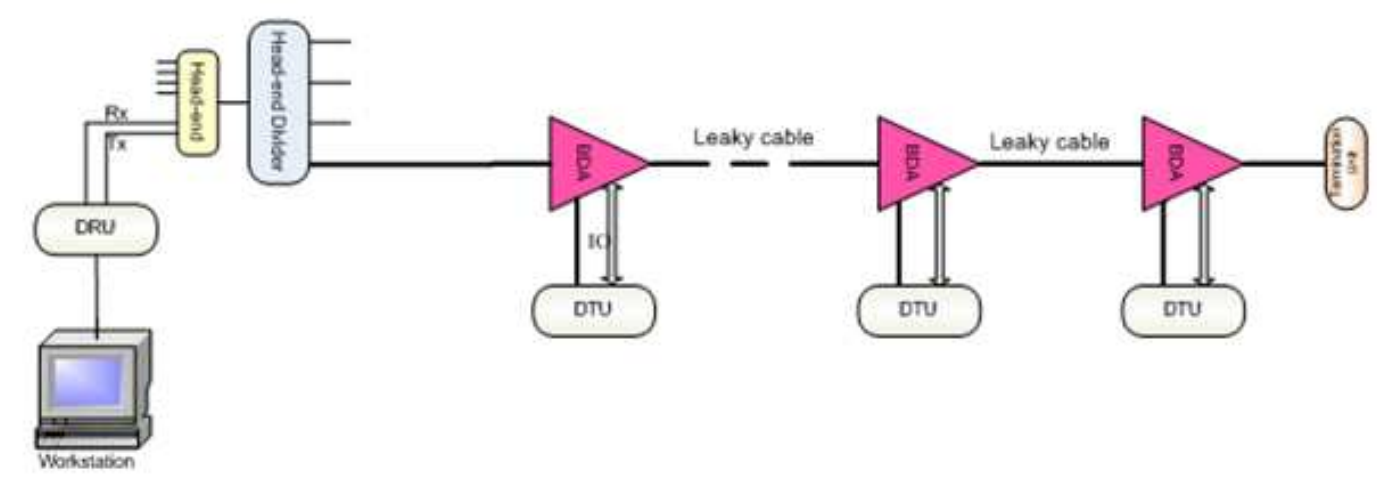

Figure 3.1: DTU Connected to BDA in Leaky Feeder Network

\subsection{Hardware Architecture}

It starts in the control room where the DRU have to be added to the already existing leaky feeder headend. The DTUs should be connected to the nodes to be monitored underground. Communication between DRU and DTUs happens through the already existing leaky cable in which some channels at specific frequencies are reserved for communication.

\subsubsection{Diagnostic Receiver Unit (DRU)}

DRU is then seen as the headend control device handling the communication, acquiring data, and exchanging commands with the DTU device. The acquired data 
is safely stored in a database further processed and analyzed by the operator. The DRU is controlled by computer in the control room run by the DSP.

DRU consists of four components:

- RF Modem: It is an RF transceiver interfacing the leaky cable responsible for communication with DTU over the leaky cable.

- Microcontroller: The microprocessor oversees all DRU activities such as packet handling and configuration.

- Memory: It is responsible for storing collected data.

- User interface: This is the interface between DRU and database/server.

DRU architecture is shown in Figure 3.2, and DRU specifications are stated in Table 3.1.

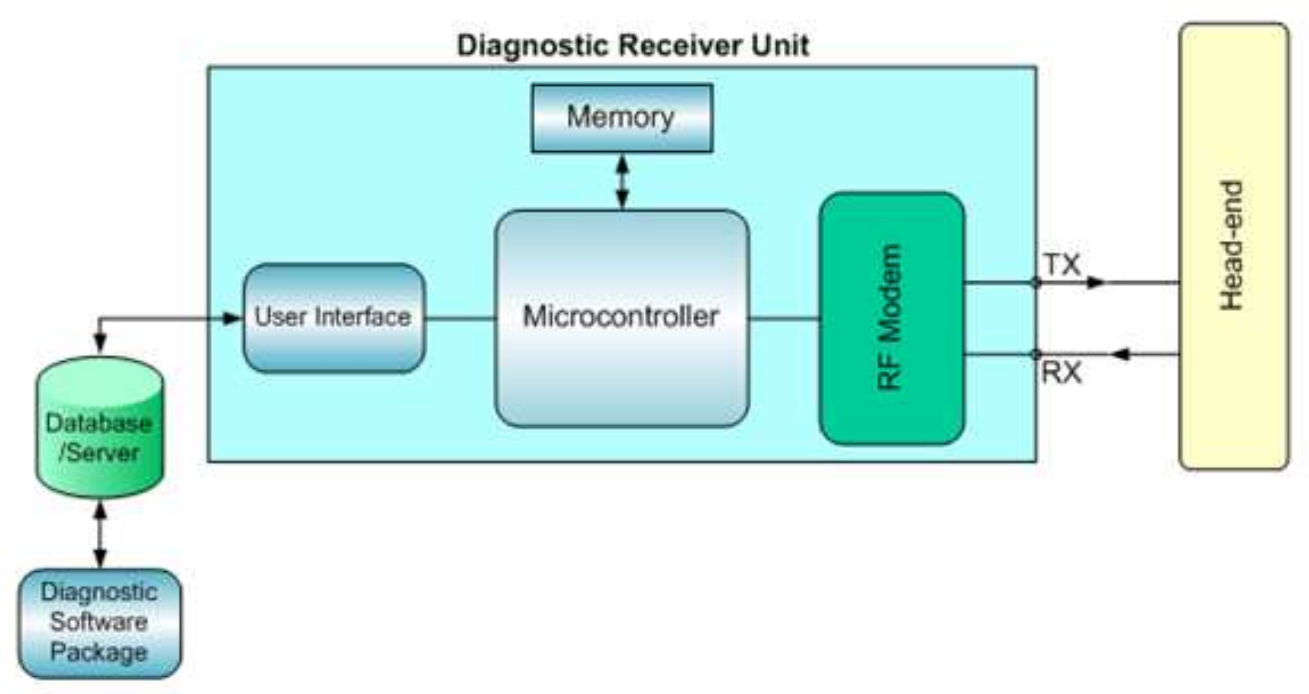

Figure 3.2: DRU Architecture 
Table 3.1: DRU Specifications

\begin{tabular}{|l|l|l|}
\hline \multirow{5}{*}{ Physical } & Construction & Rack mount enclosure \\
& Enclosure & Steel enclosure/ panel \\
& Weight & $<\mathrm{Kg}$ \\
& Connectors & RF Input/Output: BNC jack \\
& & connector Uplink/downlink Channel: \\
& & BNC jack connector \\
\hline \multirow{2}{*}{ Environmental } & Temperature & -40 to $+60 \mathrm{C}(-40$ to $+140 \mathrm{~F})$ \\
& Humidity & $10 \sim 98 \%$ \\
\hline \multirow{5}{*}{ Electrical } & Input Voltage & $12 \mathrm{~V}$ \\
& Current Consumption & $<100 \mathrm{~mA}$ \\
& Power Consumption & $<1.2 \mathrm{Watt}$ \\
\hline \multirow{5}{*}{ Receiver } & Frequency & $172-175 \mathrm{MHz}$ \\
& Modulation Type & FSK \\
& Data Format & Asynchronous burst packet-NRZ \\
& Data Rate & $19.2 \mathrm{KBaud}$ \\
& Impedance & $50 \Omega$ \\
\hline \multirow{5}{*}{ Transmitter } & Frequency & $155-158 \mathrm{MHz}$ \\
& Modulation Type & FSK \\
& Data Format & Asynchronous burst packet-NRZ \\
& Data Rate & $19.2 \mathrm{KBaud}$ \\
\hline \multirow{2}{*}{ Diagnostic } & Impedance & $50 \Omega$ \\
\hline & Green LED & Power Indicator \\
& Red LED & General Fault \\
\hline
\end{tabular}

In transmit mode, the microcontroller receives the command from the database / server package through the user interface. It processes the received command and generates the corresponding packet and passes it to RF modem to be transmitted over leaky cable.

In receive mode, the microcontroller looks for valid RF-packets. When one is received, the data contained within the RF-packet is extracted/decoded and forwarded to user interface. The data is stored in the database to be accessed later by operator for further analysis. 


\subsubsection{Diagnostic Transponder Unit (DTU)}

DTU acts as a tunable end-of-line monitor, which measures and manipulates electrical parameters of interest. It is controlled from the headend and serves as a troubleshooting tool for remote diagnostics. It assists the operator in pinpointing the location and cause of problems in the system. DTU can also be programmed to change the operating point of the attached devices or to perform automated tests on user-defined schedules where the operator does not need to be present. It enables ventilation on demand as well.

DTU consists of five major components:

- RF Modem: It is an RF transceiver responsible for communication with DRU over the leaky cable.

- Microcontroller: The microcontroller oversees all DTU activities and is responsible for all firmware in the node.

- Memory: It is responsible for storing collected data from the corresponding device.

- Data interface: This is the communication interface between DTU and attached device.

- Configuration interface: It is used for local access to DTU.

DRU architecture is shown in Figure 3.3, and DRU specifications are stated in Table 3.2 . 


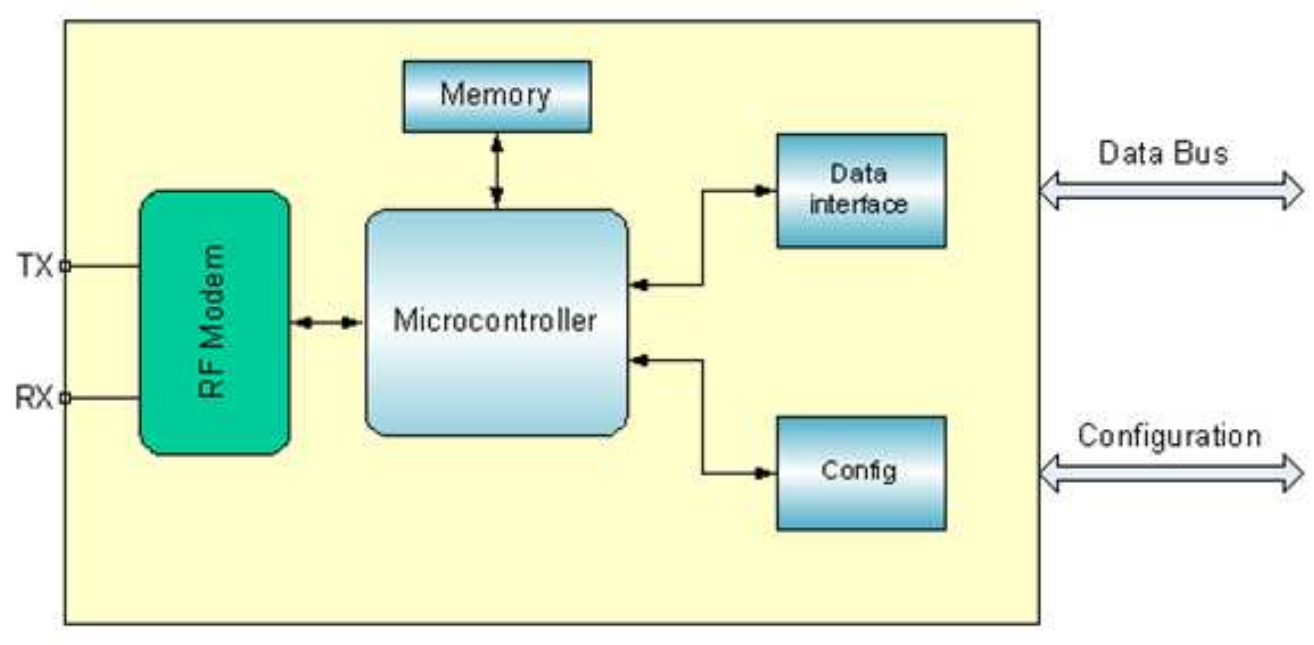

Figure 3.3: DTU Architecture

Via data bus, there is a two way communication between the micro-controller and the attached device. The microcontroller acquire data from the device, and depending on the traffic, it may store it in memory (for transmission at later time) or pass it to RF modem to be transmitted to DRU over the leaky cable. The DTU can also accept a command from DRU to change parameters of attached device. As the interface to leaky cable, the RF modem receives the command signal. The microcontroller decodes the message and performs the task on corresponding device. The task could be report/change of a parameter of corresponding device.

To aid in installation, the DTU have the connection for local access and configuration. This allows the technicians to configure the DTU on site. Through this bus the DTU can be reconfigured as well as through remote configuration using DRU. 
Table 3.2: DTU Specifications

\begin{tabular}{|l|l|l|}
\hline \multirow{5}{*}{ Physical } & Construction & Rack mount enclosure \\
& Enclosure & Steel enclosure/ panel \\
& Weight & $<\mathrm{Kg}$ \\
& Connectors & RF Input/Output: BNC jack \\
& & connector Uplink/downlink Channel: \\
& & BNC jack connector \\
\hline \multirow{2}{*}{ Environmental } & Temperature & -40 to $+60 \mathrm{C}(-40$ to $+140 \mathrm{~F})$ \\
& Humidity & $10 \sim 98 \%$ \\
\hline \multirow{5}{*}{ Electrical } & Input Voltage & $12 \mathrm{~V}$ \\
& Current Consumption & $<100 \mathrm{~mA}$ \\
& Power Consumption & $<1.2 \mathrm{Watt}$ \\
\hline \multirow{5}{*}{ Receiver } & Frequency & $155-158 \mathrm{MHz}$ \\
& Modulation Type & FSK \\
& Data Format & Asynchronous burst packet-NRZ \\
& Data Rate & $19.2 \mathrm{KBaud}$ \\
& Impedance & $50 \Omega$ \\
\hline \multirow{5}{*}{ Transmitter } & Frequency & $172-175 \mathrm{MHz}$ \\
& Modulation Type & FSK \\
& Data Format & Asynchronous burst packet-NRZ \\
& Data Rate & $19.2 \mathrm{KBaud}$ \\
\hline \multirow{2}{*}{ Diagnostic } & Impedance & $50 \Omega$ \\
\hline & Green LED & Power Indicator \\
& Red LED & General Fault \\
\hline
\end{tabular}

\subsubsection{Diagnostic Software Package (DSP)}

DSP is the point of connection between operator and the system. The diagnostic network can be monitored and analyzed using this software. All command and automated task are initiated by DSP. An automated task can be initiated to monitor/modify some parameters in the leaky cable network. Operator can also perform a spontaneous task such as monitoring a specific device or changing the operating point of a device. In term of functionality, the DSP consists of three main parts:

1. Graphical user interface (GUI) 


\section{Analysis capability}

\section{Database management}

In general, the DTU will acquire data periodically from the node connected to it, whether it is amplifier, PSU, fan, or sensor through the data interface shown in Figure 3.3. The data will be stored in the DTU's memory till it is requested by DRU. DRU can also send configuration messages to any node through the DTU's microprocessor. Furthermore, DRU is able to change RF gain of any amplifier, switch PSU on and off, increase or decrease fan speed all in its normal mode of operation.

Another operational mode is the self configuration which will be mostly used when starting up the system or when changes occur to the system layout, i.e, expanding the mine or shutting some sections in it. Two pilot signals with known values are sent from the DRU to every DTU, and the other at the time of to estimate the distance and losses between DRU and every node in the system. Interrupt is the third mode of operation. In that mode DTU nodes are able to interrupt the DRU and transmit to it important alarms, i.e, when sensors detect a high toxic gas level in the air it should inform the DTU connected to it. The DTU, in turns, interrupts the DRU process asking for immediate reaction. DRU orders DTU to switch the fans on in order to overcome the gases level in the air.

Amplifiers, PSU, fans, and sensors can interrupt the DRU at any time but according to priority. Due to the importance of ventilation, which is the mine's breathing system [20] and has a direct effect on miners' health and safety, we assigned priority 
levels. Interrupts coming from fans and sensors are given the highest priority. Following this, signals coming from amplifiers have precedence over PSU interrupts. In our protocol we use the interrupt mode and the priority levels to implement ventilation on demand.

All of the above modes are fully accessible by the operator in the control room. Via the simple DSP the operator can fully control the diagnostic system; poll nodes to read their data, poll a specific node, change the configuration of some nodes, and troubleshoot any network problems.

\subsection{Communication Protocol}

A similar protocol is the RFID communication protocol. There are several RFID communication protocols that are already being used [17, 12, 10, 3]. Comparing between these protocols and building about their results we made our diagnostic system communication protocol.

The diagnostic system protocol is based on point-to-point communications. There is a single shared channel which is the leaky feeder. The communication will happen between DRU and one DTU at a time. There are four distinct RF bands in the leaky feeder system; these bands are required to provide RF paths for voice and data communication [7].

In our protocol we consider the voice bands only. We use one voice channel for the upstream and one voice channel for the downstream to carry the messages. Particulars for the band include: Downstream voice band of 155-158 MHz, upstream voice band of $172-175 \mathrm{MHz}$, and the bandwidth of the channel is $25 \mathrm{KHz}$. Frequency 
Table 3.3: Communication Protocol Specifications

\begin{tabular}{|l|l|}
\hline Data Rate & $19.2 \mathrm{Kbps}$ \\
\hline Bandwidth & $25 \mathrm{KHz}$ \\
\hline Downstream Frequency & $155-158 \mathrm{MHz}$ (tunable) \\
\hline Upstream Frequency & $172-175 \mathrm{MHz}$ (tunable) \\
\hline Modulation & FSK \\
\hline
\end{tabular}

\begin{tabular}{|c|c|c|c|c|c|c|c|}
\hline $\begin{array}{c}\text { Preamble } \\
\text { bits }\end{array}$ & $\begin{array}{c}\text { Source } \\
\text { Address }\end{array}$ & $\begin{array}{c}\text { Destination } \\
\text { Address }\end{array}$ & Control & Priority & Reserved & Data & CRC \\
\hline
\end{tabular}

Figure 3.4: General Message Format

modulation is preferred in the leaky feeders due to its continuous nature in the time domain, therefore, we used FSK modulation technique.

This Diagnostic system doesn't require a high data rate since it is not a time sensitive application, so we used $19.2 \mathrm{Kbps}$. Table 3.3 shows the communication protocol specifications. The protocol runs in the following three different modes: self configuration, normal mode (read-write), and interrupt. Self configuration and normal mode are initiated by the operator in the control room while the interrupt is initiated automatically from any DTU. General message format is shown in Figure 3.4 and consists of the following fields.

- Preamble bits: indicates the start of the frame and used for synchronization between transmitter and receiver. Formed of 16 bits of alternating ones and zeros 1010101010. 
- Source address: 8 bits indicating the address of the transmitter i.e. system can have 256 nodes in total.

- Destination address: 8 bits indicating the address of the receiver i.e. total of 256 nodes can be supported.

- Control: 8 bits

- Bits 0-1: Device type: PSU=00, Amplifier=11, fan and sensor=10

- Bits 2-4: Represents the type of the message as shown in Table 3.4.

Table 3.4: Bits 2-4 of the Control Field

\begin{tabular}{|l|l|}
\hline Message & Value \\
\hline$R R E S$ & 000 \\
\hline$A C K \_S E T \_D$ & 111 \\
\hline$S E T \_D$ & 100 \\
\hline$R E Q$ & 010 \\
\hline$W R I T E \_D A T A$ & 001 \\
\hline$R E A D \_D A T A$ & 011 \\
\hline$A C K$ & 101 \\
\hline$I N T$ & 110 \\
\hline
\end{tabular}

- Bit 5-6: set RF killer/ current RF killer status, RF killer off=00, RF killer on $=11$.

- Bit 7: reserved

- Priority: 2 bits indicate the importance of the message.

- Low priority $=00$

- Normal priority-PSU $=01$

- Medium priority-amplifiers $=10$ 
- High priority-fans and sensors $=11$

- Reserved: 8 bits reserved for future use

- Data: It is the payload.

For normal mode-read cycle: Data consists of nine bytes containing information about amplifier, and in case of PSU it is eight bytes of information, and four bytes of information in case of fans and sensor. For normal mode-write cycle: Data represents the information required to program the node. Four bytes are used in case of amplifier and three bytes for the case of PSU and fans and sensors.

- CRC: 16 bits Cyclic Redundancy Check, Preamble bits are not used in the calculation of the CRC bytes. Using the code word.

$$
C(x)=x^{16}+x^{15}+x^{2}+1
$$

\subsubsection{Self Configuration}

This mode must be run after commissioning the network and whenever any changes happen to the mine i.e. adding a new section or closing a current section in the mine. This mode allows detecting the network layout in terms of number of nodes, their type and location as well as detecting the losses between nodes. This is made possible by sending pilot signals with known values from DRU to each DTU.

On the downstream the DRU will send two pilot signals which will suffer attenuation by the time they reach the DTU node. From this attenuation and using complex 
algorithm DTU can calculate the slope and estimate the distance between itself and the DRU, losses in between, and the existence of any splitters between them as well. Figure 3.5 shows pilot signals at DRU and DTU.

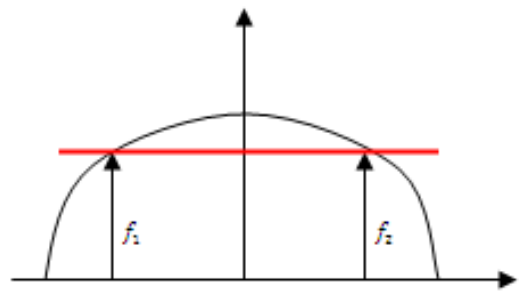

Pilot signal generated at DRU

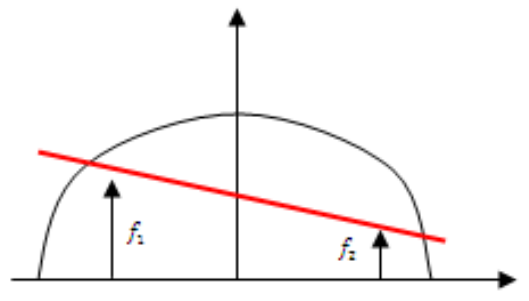

Pilot signal received at DTU

Figure 3.5: Self Configuration Pilot Signal at DRU and the Attenuated Version at DTU

RF killer should be added to the output of all downstream DTU's as it will stop the pilot signal from propagating further till the losses and distance between the DRU and DTU communicating to it are calculated. Figure 3.6, shows the RF killer in the downstream direction. DTU's will have a flag "D"

- $\mathrm{D}=0 \quad$ RF killer off. i.e. signals can pass

- $\mathrm{D}=1 \quad$ RF killer on. i.e. signals are blocked

Self configuration methodology:

- DRU broadcasts the message (SET_D) to all nodes to set RF killer on.

- RF killer stops the propagation of signals to any further nodes.

- DRU start communicating with the first DTU by sending two pilot signal with known values at frequency $\left(f_{1}\right)$ and frequency $\left(f_{2}\right)$. 


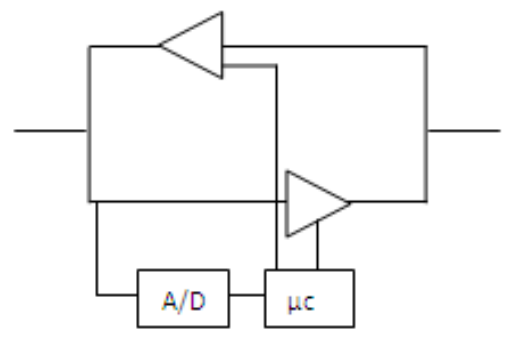

No RF killer

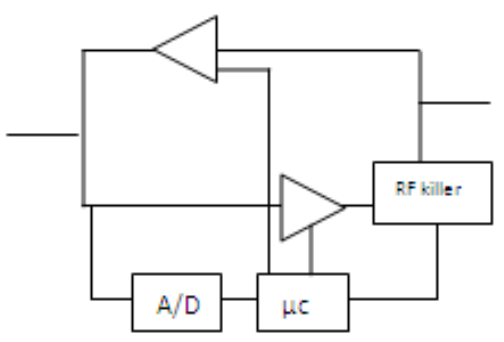

RF killer on the down stream

Figure 3.6: RF killer in place on the downstream

- DTU records the values of the pilot signal received and sends them back to the DRU along with the node type using the (RRES) message. Knowing the value of the sent pilot signal and the received pilot at DTU, DRU will run algorithm to estimate distance between the nodes and losses in the leaky. This information is sent to the amplifier to set the gain.

- DRU sends a message to acknowledge and set the RF killer off in order to start communicating with the next node using the message (ACK_SET_D).

- DRU updates its database by adding all information about this node.

- DRU repeats the same sequence with the following nodes till all nodes are done.

System loss in each cable section, $L_{n, m}$, can be calculated by

$$
L_{n, m}=C L_{n, m}\left(x_{n}, f_{m}\right)+I L_{n}
$$

$L_{n, m}$ : Total system loss in one section of cable, $n$, at frequency band $m$, this is the value detected at DTU.

$C L_{n, m}$ : Longitudinal cable loss in one section, its function of cable length $x_{n}$ and 
frequency $f_{m}$.

$I L_{n}$ : Insertion loss installed on section $n$.

$n$ : Number of cable section.

$m$ : Band number in the system.

$C L_{n, m}$ : The cable loss per meter at certain frequency, which can be obtained from the cable manufacturer's data sheet or by sweep test.

Considering a section, $n$, of the leaky feeder cable where two pilot signals are sent from DRU to DTU at frequencies $f_{1}$, and $f_{2}$, respectively. Losses in this section at both frequencies are:

$$
\begin{aligned}
& L_{n, 1}=\text { Pilot }_{1}-R x_{n, 1}=C L_{n, 1}\left(x_{n}, f_{1}\right)+I L_{n} \\
& L_{n, 2}=\text { Pilot }_{2}-R x_{n, 2}=C L_{n, 2}\left(x_{n}, f_{2}\right)+I L_{n}
\end{aligned}
$$

$R x_{n, m}$ : Pilot value as received and recorded at DTU cable section, $n$, at frequency $m$.

Obtaining cable loss $C L_{n, m}$ at $f_{1}$ and $f_{2}$ using data sheet or sweep test we can develop a relation between them called $R_{d}$. Figure 3.7 shows the data sheet that we used in our calculations.

$$
R_{d}=\frac{C L_{n, 1}}{C L_{n, 2}}
$$

From equation 3.3 .3 and 3.3.5

$$
I L=L_{n, 1}-R_{d} \cdot C L_{n, 2}\left(x_{n}, f_{2}\right)
$$




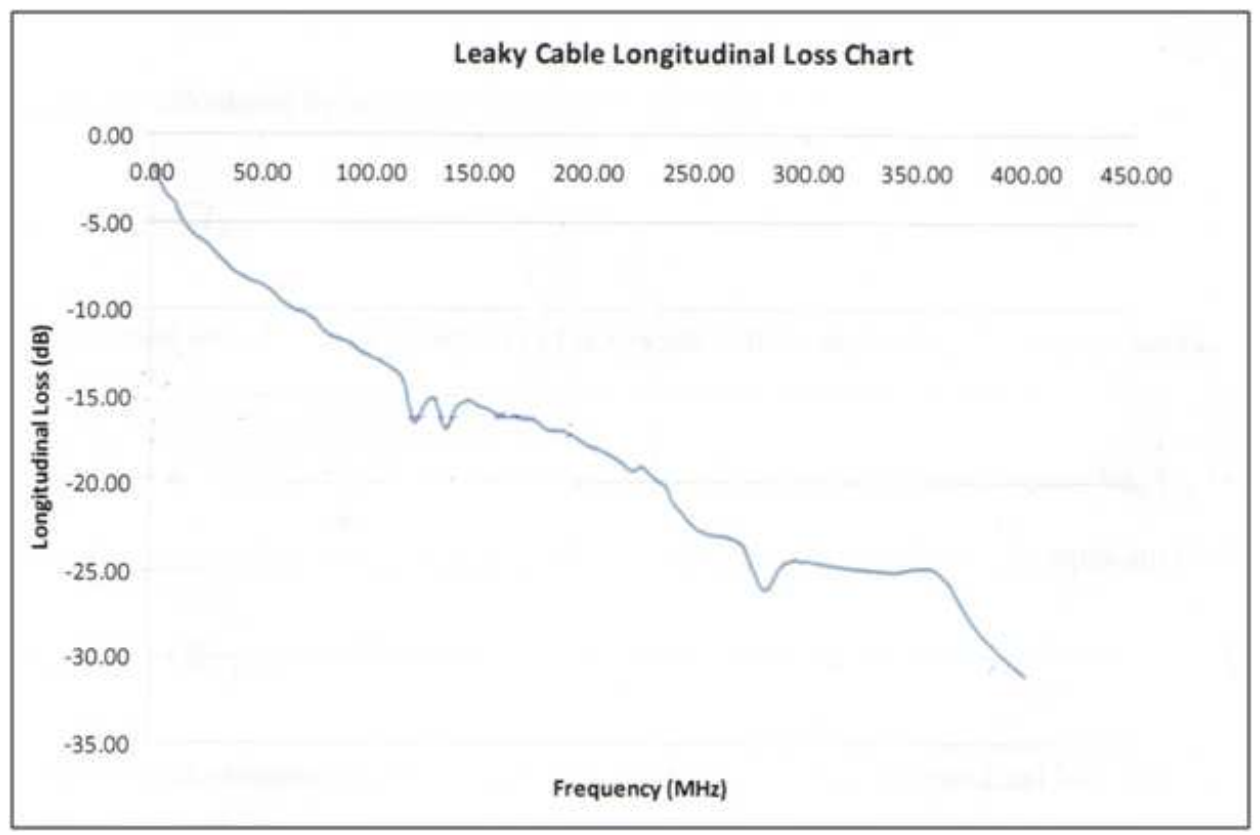

Figure 3.7: Leaky Cable Longitudinal Loss Chart

using equations 3.3.4 and 3.3.6

$$
\begin{gathered}
L_{n, 2}=C L_{n, 2}\left(x_{n}, f_{2}\right)+L_{n, 1}-R_{d} \cdot C L_{n, 2}\left(x_{n}, f_{2}\right) \\
L_{n, 2}=C L_{n, 2}\left(x_{n}, f_{2}\right) \cdot\left[1-R_{d}\right]+L_{n, 1} \\
C L_{n, 2}\left(x_{n}, f_{2}\right)=\frac{L_{n, 2}-L_{n, 1}}{\left[1-R_{d}\right]}
\end{gathered}
$$

and, from equation 3.3.4

$$
I L=L_{n, 2}-C L_{n, 2}\left(x_{n}, f_{2}\right)
$$

Thus, using equations 3.3.8, 3.3.9, and 3.3.10

$$
I L=L_{n, 2}-\frac{L_{n, 2}-L_{n, 1}}{\left[1-R_{d}\right]}
$$

Insertion loss $(I L)$ is a result of splitters installed along the leaky feeder cable. These splitters cause a fixed signal drop by $3.5 \mathrm{~dB}$ per splitter. Thus, number of splitters 


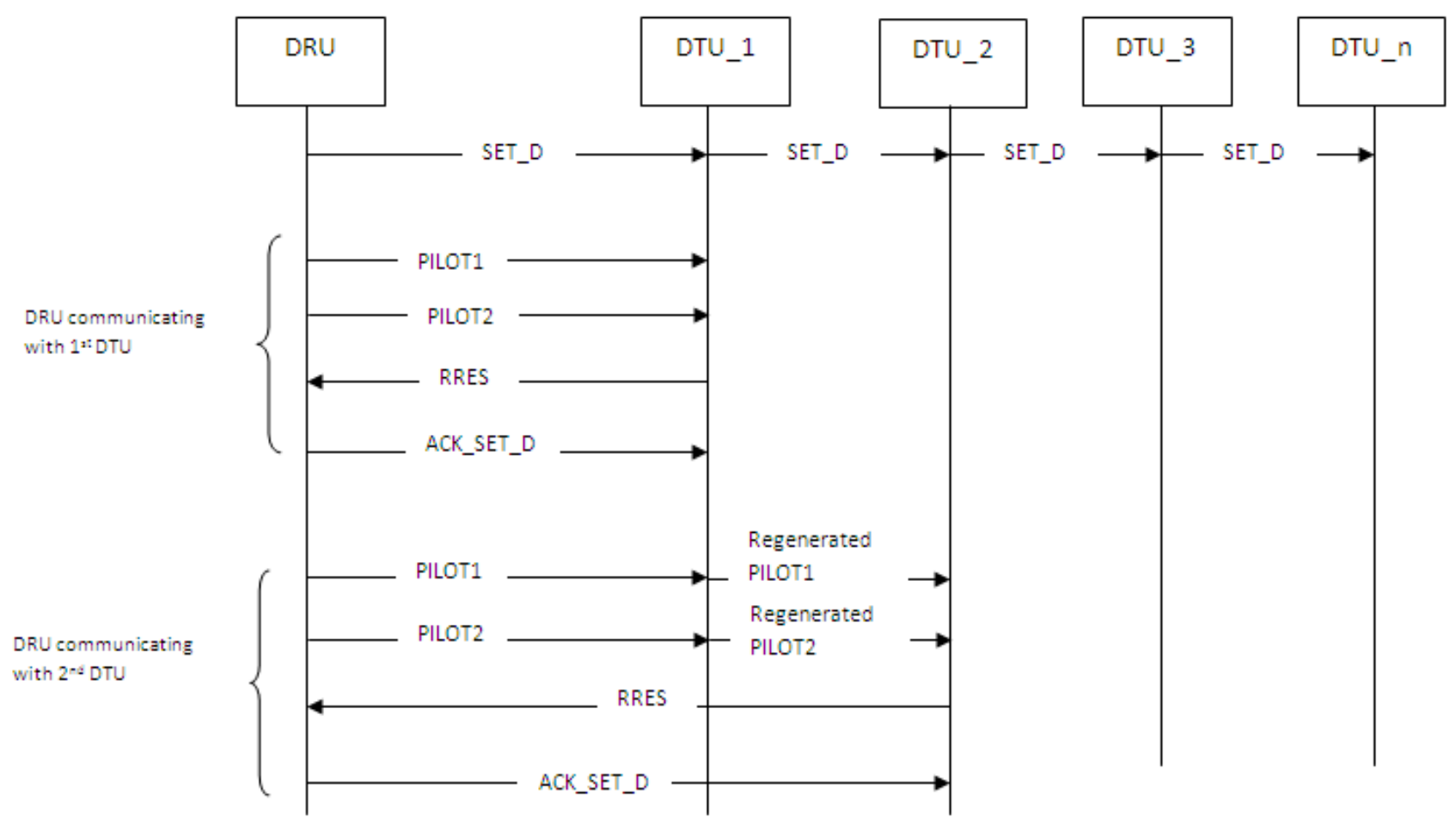

Figure 3.8: Self Configuration Message Sequence

can be given by

$$
\text { numberof splitters }=\frac{I L}{3.5}
$$

Length of each section is calculated using

$$
\text { Cablelength }(\text { persection })=\frac{C L_{n, 2}\left(x_{n}, f_{2}\right)}{C L_{n, 2}}
$$

Running this algorithm detects the number and type of nodes in each section of the network, and also the length, number of splitters, and losses in each section of the leaky feeder cable. Using this information it is easy to recognize the network layout and visualize it in a GUI using the DSP at the control room above ground. Self configuration message sequence is shown in Figure 3.8. 


\begin{tabular}{|l|l|l|l|}
\hline Preamble bits & Control & Reserved & CRC \\
\hline
\end{tabular}

Figure 3.9: SET_D Message Format

The messages sent and received in the self configuration mode are as follow: SET_D message

Purpose: broadcast message to all DTU's setting their RF killer on. Stop pilot signal from propagating until communication between DRU and that specific DTU is over. Message format is shown in Figure 3.9

- Preamble: 16 bits for synchronization and indicating the start of the frame

- Control: 8 bits

- Bits 0-1: Not used

- Bits 2-4: Represents the type of the message as shown in Table 3.4. Here, it is a SET_D message with the value $\mathrm{SET}_{-} \mathrm{D}=100$

- Bit 5-6: RF killer setting, set flag D: (RF killer off $\mathrm{D}=0)=00,(\mathrm{RF}$ killer on $\mathrm{D}=1)=11$. In this case it's RF killer on $=11$

- Bit 7: reserved

- Reserved: 8 bits reserved for future use

- CRC: 16 bits check sum. Preamble bits are not included in the calculation

RRES: Route Response message 
Purpose: sending the values of Pilot 1 and pilot 2 as received by DTU, also sending status of air and fan sensors.

\begin{tabular}{|l|l|l|l|l|l|}
\hline Preamble & PILOT Reply & Status & Control & Reserved & CRC \\
\hline
\end{tabular}

Figure 3.10: RRES Message Format

- Preamble: 16 bits for synchronization and indicating the start of the frame

- PILOT Reply: 2 bytes representing the recorded loss. byte 1: total loss at $f_{1}$, byte 2 : total loss at $f_{2}$

- Status: 4 bits represent sensor and fan status, 2 bits for each

- Bits 0-1: sensor status: Sensor off/ no sensor $=00$, normal air quality $=$ 01, medium air quality (acceptable value of pollutants) $=10$, high level of pollutants $=11$

- Bits 2-3: Fan status: Fan off/no fan=00, fan on, low speed=01, fan on medium speed $=10$, fan on high speed $=11$

- Control: 8 bits

- Bits 0-1: device type: $\mathrm{PSU}=00$, Amplifier $=11$, fan and sensor $=10$

- Bits 2-4: Represents the type of the message as shown in Table 3.4. Here, it is a RRES message with the value $\mathrm{RRES}=000$ 
- Bit 5-6: current RF killer status, (RF killer off $\mathrm{D}=0)=00$, ( RF killer on $\mathrm{D}=1)=11$. In this case it's RF killer on $=11$

- Bit 7: reserved

- Reserved: 8 bits reserved for future use

- CRC: 16 bits check sum. Preamble bits are not included in the calculation

\section{ACK_SET_D message}

Purpose: DRU acknowledge the recipient of the data, and turning the RF killer off to allow pilot signal propagate further and poll different DTU's.

\begin{tabular}{|l|l|l|l|}
\hline Preamble & Control & Reserved & CRC \\
\hline
\end{tabular}

Figure 3.11: ACK_SET_D Message Format

- Preamble: 16 bits for synchronization and indicating the start of the frame.

- Control: 8 bits

- Bits 0-1: device type: PSU $=00$, Amplifier $=11$, fan and sensor $=10$

- Bits 2-4: Represents the type of the message as shown in Table 3.4. Here, it is a ACK_SET_D message with the value ACK_SET_D=100

- Bit 5-6: RF killer setting, set flag D: $(\mathrm{RF}$ killer off $\mathrm{D}=0)=00,(\mathrm{RF}$ killer on $\mathrm{D}=1)=11$. In this case it's RF killer off $=00$

- Bit 7: reserved 
- Reserved: 8 bits reserved for future use

- CRC: 16 bits check sum. Preamble bits are not included in the calculation

In order to overcome software problems like hanging, and malfunctioning we propose a watch-dog-like program at the microprocessor of each node. After the node transmits a message it waits for a Maximum Time to Wait (MTW). If it does not receive any response then the message is retransmitted and the node waits for MTW. If a response is not received within this second MTW we assume a problem occurred and an alarm is sent to the control room.

Figure 3.12, shows the self configuration operation mode. DRU and DTU subroutines are shown at Figures 3.13 and 3.14, respectively. 


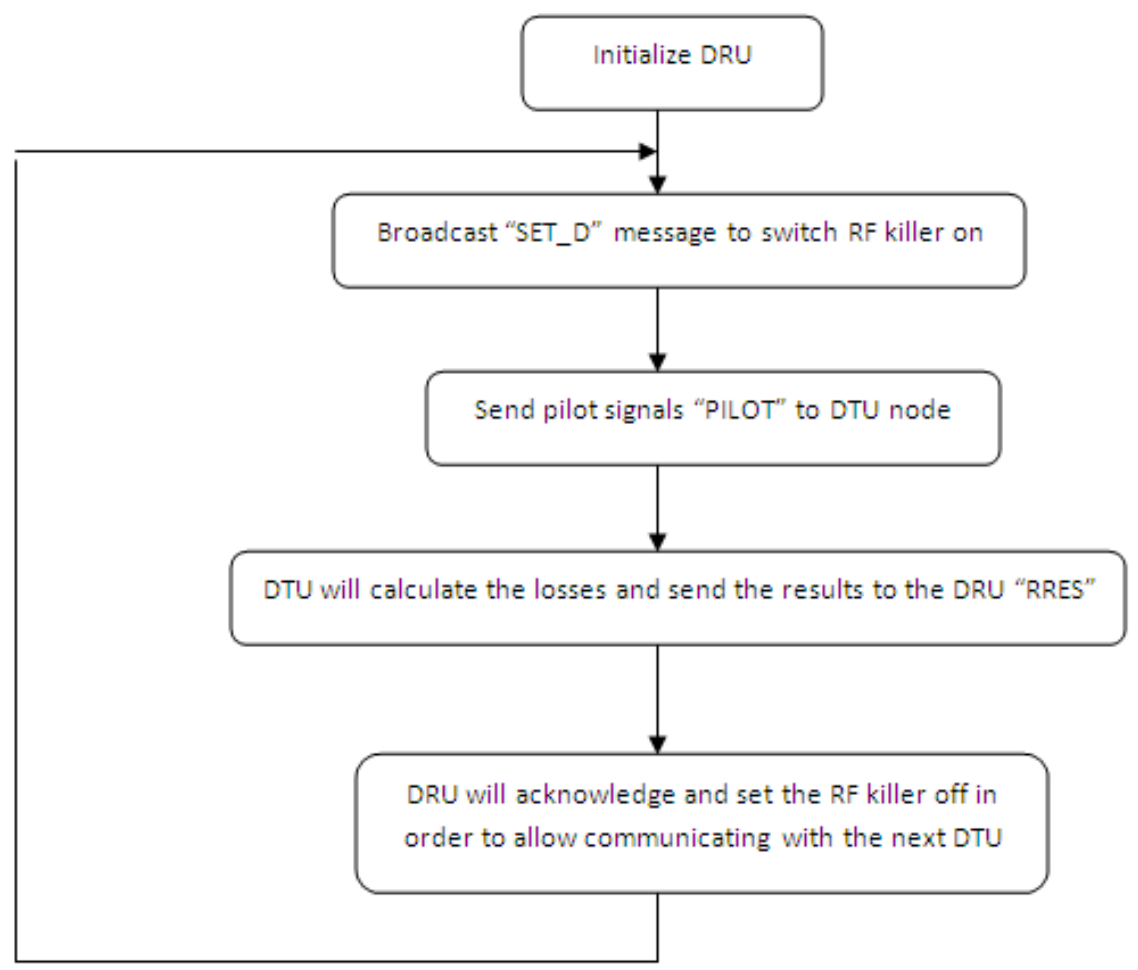

Figure 3.12: Self Configuration Operation Mode 


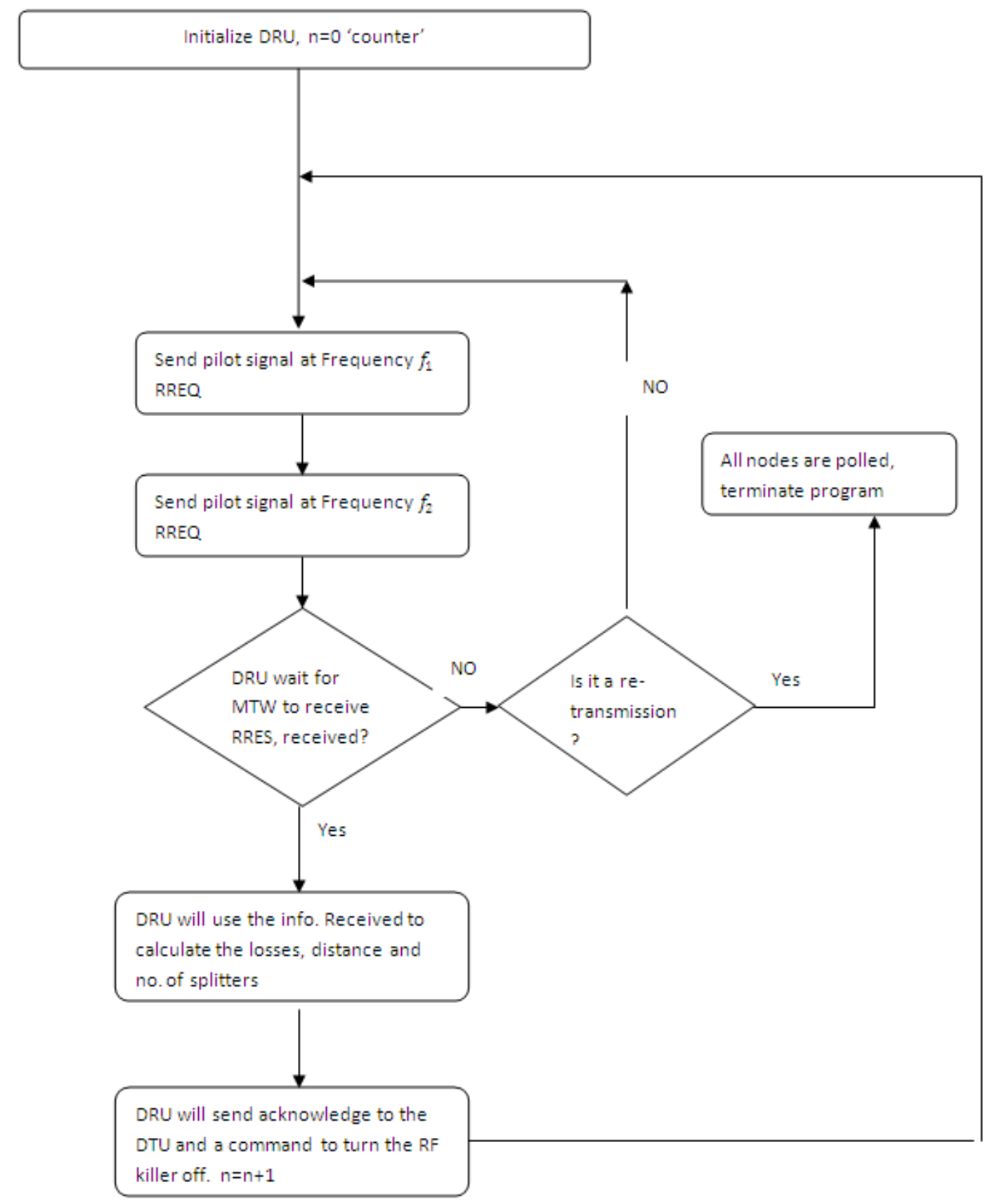

Figure 3.13: DRU Flowchart 


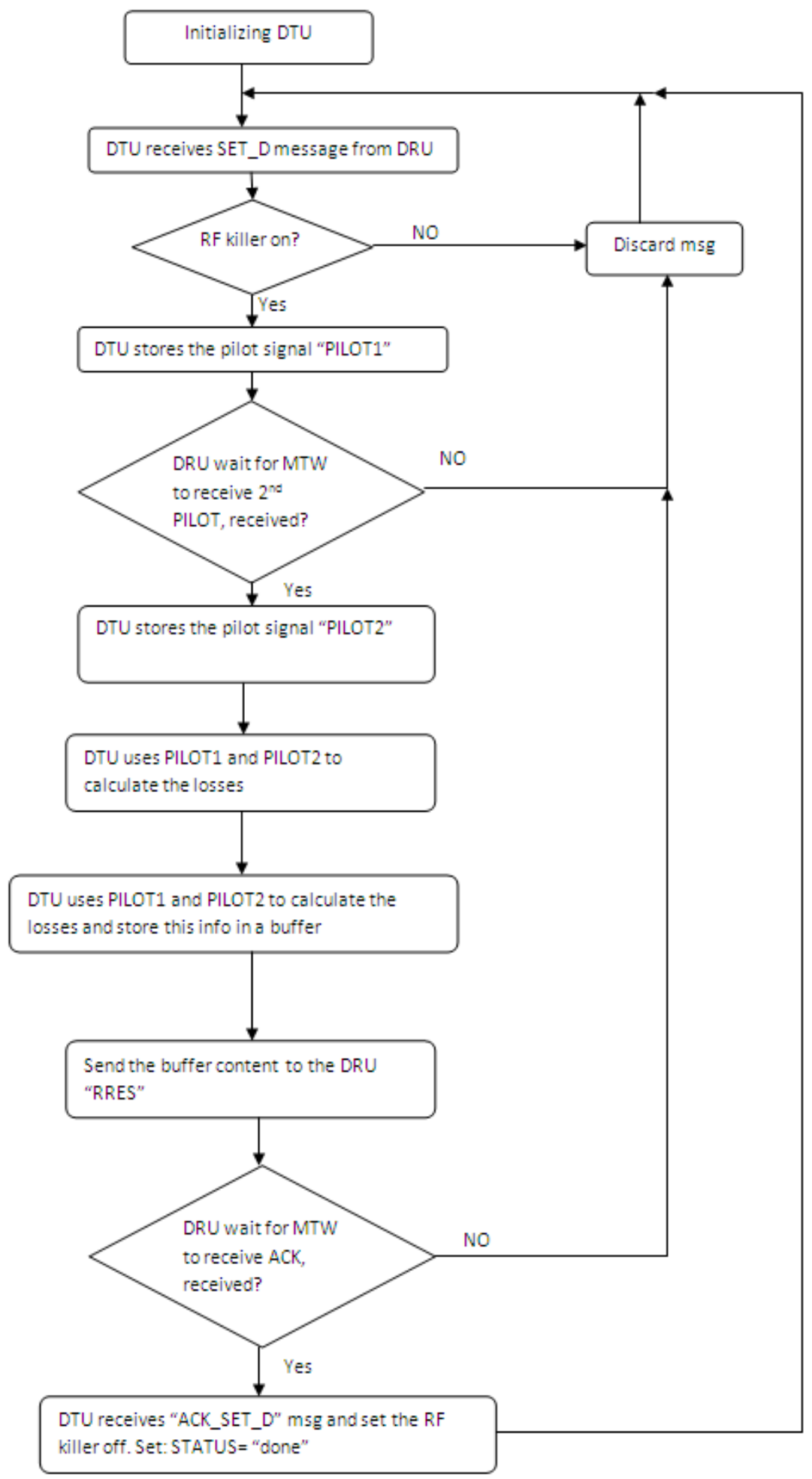

Figure 3.14: DTU Flowchart 


\subsubsection{Normal Mode}

This mode can do both read and write cycles. It enables DRU to send writing data to any DTU to change parameters of attached amplifier, PSU, fans, and sensors. It can also perform read cycle which enables DRU to poll all DTU's in sequence and read data stored into them which was extracted earlier from amplifier, PSU, fans, and sensors attached.

This mode will not work unless the self configuration mode runs first because it requires information about the number of nodes and their type.

The two cycles are:

\section{Write Cycle}

Write cycle occurs when the operator needs to program a node without sending a technician underground. The commands are sent in the data field. For amplifiers the DRU can program the amplifier gain for the upstream and downstream voice channels as well as the amplifier gain for the data channel. In case of PSU the DRU can switch the power on or off and switch between primary battery and pack up battery. For fans and sensors the DRU is able to switch the fan on or off in addition to increasing or decreasing fan speed.

As shown in Figure 3.15, the write cycle happens as follows:

- DRU identifies the DTU it wishes to program and starts communicating to it by sending (WRITE) message, the data field carries the information to be 
programmed.

- DTU receives the message, extracts the data, and using the micro processor it will program the attached node. When done it will reply back with (ACK) message to the DRU.

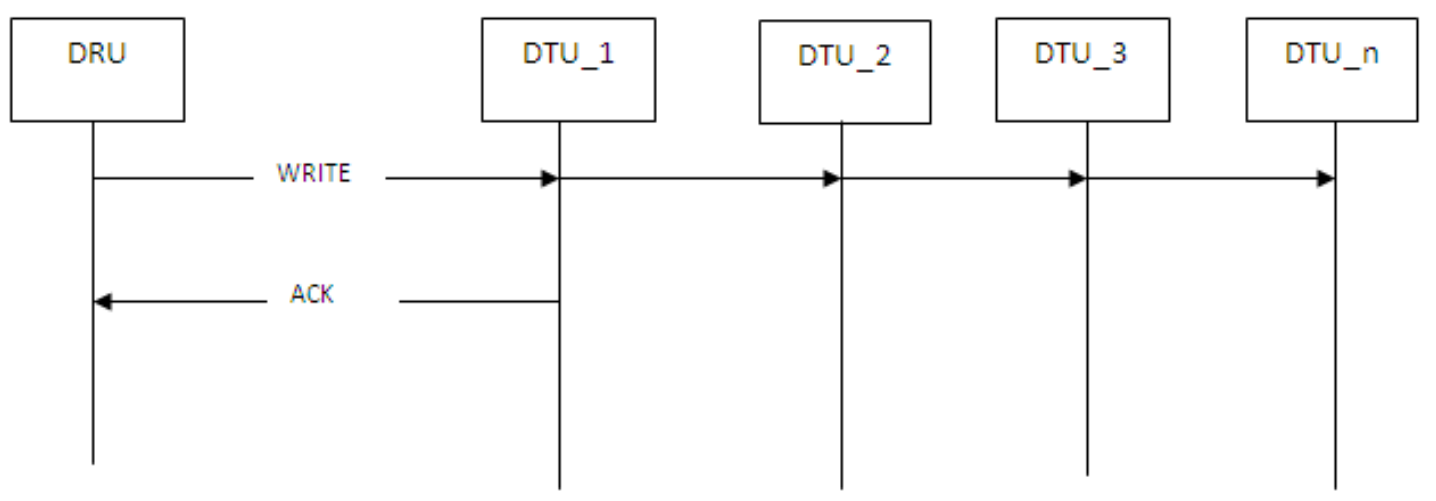

Figure 3.15: Normal Mode- Write Cycle Message Sequence

The messages sent and received in the Normal mode. Write cycle are as follow:

\section{WRITE message}

Purpose: write data to a specific DTU. i.e. change parameters of amplifier, PSU, Fan and Sensor.

- Preamble: 16 bits for synchronization and indicating the start of the frame

- Source address: 8 bits indicating the message origin. Here it will be DRU address. 8 bits means that the system supports maximum 256 nodes. 


\begin{tabular}{|c|c|c|c|c|c|c|c|}
\hline $\begin{array}{c}\text { Preamble } \\
\text { bits }\end{array}$ & $\begin{array}{c}\text { Source } \\
\text { Address }\end{array}$ & $\begin{array}{c}\text { Destination } \\
\text { Address }\end{array}$ & Control & Priority & DATA & Reserved & CRC \\
\hline
\end{tabular}

Figure 3.16: Format of WRITE Message

- Destination address: 8 bits indicating the receiver address. Here it will be the intended DTU. 8 bits means that the system supports maximum 256 nodes.

- Control: 8 bits

- Bits 0-1: device type: $\mathrm{PSU}=00$, Amplifier $=11$, fan and sensor $=10$

- Bits 2-4: Represents the type of the message as shown in Table 3.4. Here, it is a WRITE_DATA message with the value WRITE_DATA=001

- Bit 5-7: reserved

- Priority: 2 bits indicate the importance of the message, if more than one message sent they are held in sequence and the message with higher priority is handled first.

- Low priority $=00$

- Normal priority "default" here we will set it for PSU $=01$

- Medium priority, we will set it for amplifiers $=10$

- High priority, we will set it for fans and sensors $=11$

- Data: 4 bytes as shown in Table 3.5

- Reserved: 8 bits reserved for future use 
Table 3.5: Data Configured During the Write Cycle

\begin{tabular}{|l|l|l|l|}
\hline \multicolumn{3}{|c|}{ Write Cycle } \\
\hline & Amplifier & Battery & Fan and sensor \\
\hline Byte 1 & Amplifier Gain \#1 & ON/OFF & Fan ON/OFF \\
\hline Byte 2 & Amplifier Gain \#2 & Switch (Primary/ backup) & Increase/ decrease fan speed \\
\hline Byte 3 & Amplifier Gain \#3 & Reserved & Reserved \\
\hline Byte 4 & Reserved & - & - \\
\hline
\end{tabular}

- CRC: 16 bits check sum. Preamble bits are not included in the calculation

ACK: Acknowledge message

Purpose: DRU informs DTU that it received the message and terminates connection.

\begin{tabular}{|c|c|c|c|c|c|c|}
\hline Preamble & $\begin{array}{c}\text { Source } \\
\text { Address }\end{array}$ & $\begin{array}{c}\text { Destination } \\
\text { Address }\end{array}$ & Control & Priority & Reserved & CRC \\
\hline
\end{tabular}

Figure 3.17: Format of ACK Message

- Preamble: 16 bits for synchronization and indicating the start of the frame.

- Source address: 8 bits indicating the message origin, here it will be DTU originator address. 8 bits means that the system supports maximum 256 nodes.

- Destination address: 8 bits indicating the receiver address, here it will be DRU. 8 bits means that the system supports maximum 256 nodes.

- Control: 8 bits

- Bits 0-1: device type: PSU $=00$, Amplifier $=11$, fan and sensor $=10$ 
- Bits 2-4: Represents the type of the message as shown in Table 3.4. Here, it is a WRITE_DATA message with the value ACK=101

- Bit 5-7: reserved

- Priority: 2 bits indicate the importance of the message. If more than one message is sent they are held in sequence and the message with higher priority is handled first.

- Low priority $=00$

- Normal priority "default" here we will set it for PSU $=01$

- Medium priority, we will set it for amplifiers $=10$

- High priority, we will set it for fans and sensors $=11$

- Reserved: 8 bits reserved for future use.

- CRC: 16 bits check sum. Preamble bits are not included in the calculation.

\section{Read Cycle}

Read cycle is when the DRU is reading information from DTU. For amplifiers it will read the RF level and amplifier gain for the up and downstream voice channels and the data channel as well as reading the amplifier DC voltage and current. For PSU it will read DC and AC voltages, and DC current, temperature, hours of operation, and backup battery status. In fans and sensors; air quality, status of fan in addition to fan speed will be read. DRU will poll all DTU's in order to read their information. Figure 3.18, shows the reading operation.

- DRU contacts the first DTU by sending to it a request to read (REQ) message. 
- DTU reply back with the data stored in memory (DATA_READ) message.

- DRU acknowledges the data reception and terminates the connection in order to poll the next node using (ACK) message.

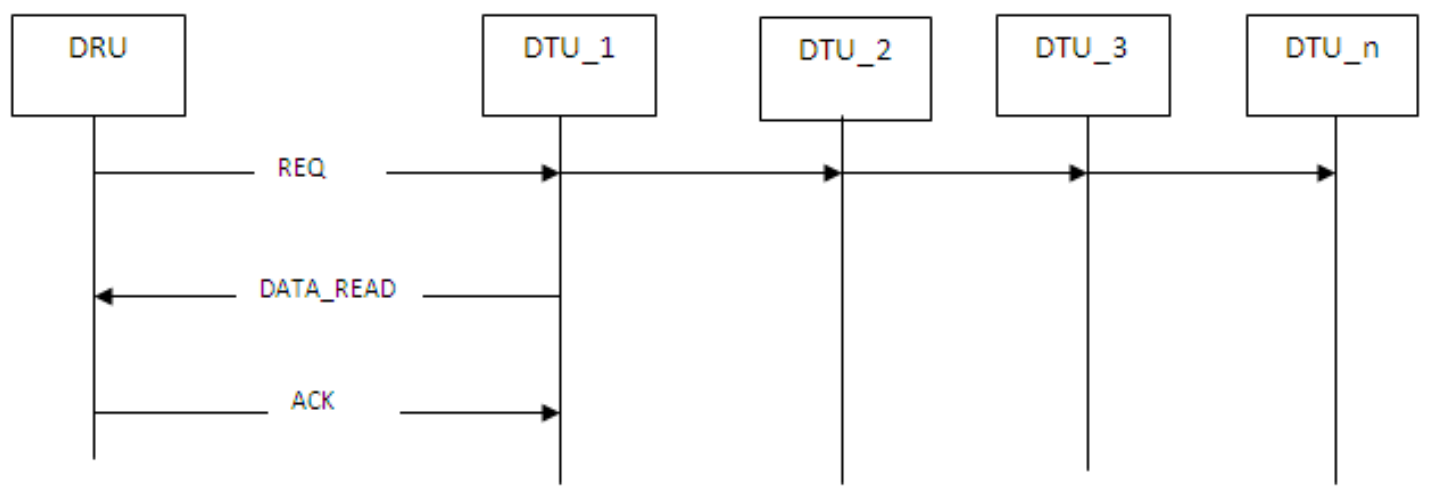

Figure 3.18: Normal Mode- Read Cycle Message Sequence

The messages sent and received in the Normal mode- Read cycle are as follow:

REQ: Request message

Purpose: request data message, sent from DRU to DTU.

\begin{tabular}{|c|c|c|c|c|c|c|}
\hline $\begin{array}{c}\text { Preamble } \\
\text { bits }\end{array}$ & $\begin{array}{c}\text { Source } \\
\text { Address }\end{array}$ & $\begin{array}{c}\text { Destination } \\
\text { Address }\end{array}$ & Control & Priority & Reserved & CRC \\
\hline
\end{tabular}

Figure 3.19: Format of REQ Message

- Preamble: 16 bits for synchronization and indicating the start of the frame.

- Source address: 8 bits indicating the message origin, here it will be DRU address. 8 bits means that the system supports maximum 256 nodes. 
- Destination address: 8 bits indicating the receiver address, here it will be DTU. 8 bits means that the system supports maximum 256 nodes.

- Control: 8 bits

- Bits 0-1: device type: PSU $=00$, Amplifier $=11$, fan and sensor $=10$

- Bits 2-4: Represents the type of the message as shown in Table 3.4. Here, it is a $R E Q$ message with the value $R E Q=010$

- Bit 5-7: reserved

- Priority: 2 bits indicate the importance of the message, if more than one message sent they are held in sequence and the message with higher priority is handled first.

- Low priority $=00$

- Normal priority "default" here we will set it for PSU $=01$

- Medium priority, we will set it for amplifiers $=10$

- High priority, we will set it for fans and sensors=11

- Reserved: 8 bits reserved for future use.

- CRC: 16 bits check sum. Preamble bits are not included in the calculation.

DATA_READ message

Purpose: read data from specific DTU. i.e. change parameters of amplifier, PSU, fan and sensor.

- Preamble: 16 bits for synchronization and indicating the start of the frame. 


\begin{tabular}{|c|c|c|c|c|c|c|c|}
\hline $\begin{array}{c}\text { Preamble } \\
\text { bits }\end{array}$ & $\begin{array}{c}\text { Source } \\
\text { Address }\end{array}$ & $\begin{array}{c}\text { Destination } \\
\text { Address }\end{array}$ & Control & Priority & DATA & Reserved & CRC \\
\hline
\end{tabular}

Figure 3.20: Format of DATA_READ Message

- Source address: 8 bits indicating the message origin, here it will be DTU originator address. 8 bits means that the system supports maximum 256 nodes.

- Destination address: 8 bits indicating the receiver address, here it will be the intended DRU. 8 bits means that the system supports maximum 256 nodes.

- Control: 8 bits

- Bits 0-1: device type: $\mathrm{PSU}=00$, Amplifier $=11$, fan and sensor $=10$

- Bits 2-4: Represents the type of the message as shown in Table 3.4. Here, it is a READ_DATA message with the value READ_DATA=011

- Bit 5-7: reserved

- Priority: 2 bits indicate the importance of the message, if more than one message sent they are held in sequence and the message with higher priority is handled first.

- Low priority $=00$

- Normal priority "default" here we will set it for PSU $=01$

- Medium priority, we will set it for amplifiers $=10$

- High priority, we will set it for fans and sensors $=11$ 
Table 3.6: Data Read During Read Cycle

\begin{tabular}{|l|l|l|l|}
\hline \multicolumn{5}{|c|}{ Read Cycle } \\
\hline & Amplifier & Battery & Fan and sensor \\
\hline Byte 1 & RF level \#1 & DC voltage & Air quality (sensor) \\
\hline Byte 2 & RF level \#2 & DC current & ON/OFF (fan) \\
\hline Byte 3 & RF level \#3 & AC voltage & fan speed \\
\hline Byte 4 & Amplifier gain \#1 & Temperature & Reserved \\
\hline Byte 5 & Amplifier gain \#2 & Hours of operation & - \\
\hline Byte 6 & Amplifier gain \#3 & Battery voltage & - \\
\hline Byte 7 & Amplifier DC voltage & Battery backup status & - \\
\hline Byte 8 & Amplifier DC current & Reserved & - \\
\hline Byte 9 & Reserved & - & - \\
\hline
\end{tabular}

- Data: 9 bytes as shown in Table 3.6

- Reserved: 8 bits reserved for future use

- CRC: 16 bits check sum. Preamble bits are not included in the calculation

\section{ACK: Acknowledge message}

Purpose: DRU informs DTU that it received the message and terminates connection. It is the same ACK in Figure 3.17, with the below specifications.

- Preamble: 16 bits for synchronization and indicating the start of the frame.

- Source address: 8 bits indicating the message origin, here it will be DTU originator address. 8 bits means that the system supports maximum 256 nodes.

- Destination address: 8 bits indicating the receiver address, here it will be DRU. 8 bits means that the system supports maximum 256 nodes.

- Control: 8 bits

- Bits 0-1: device type: PSU $=00$, Amplifier $=11$, fan and sensor $=10$ 
- Bits 2-4: Represents the type of the message as shown in Table 3.4. Here, it is a ACK message with the value ACK=101

- Bit 5-7: reserved

- Priority: 2 bits indicate the importance of the message, if more than one message sent they are held in sequence and the message with higher priority is handled first.

- Low priority $=00$

- Normal priority "default" here we will set it for PSU $=01$

- Medium priority, we will set it for amplifiers $=10$

- High priority, we will set it for fans and sensors=11

- Reserved: 8 bits reserved for future use.

- CRC: 16 bits check sum. Preamble bits are not included in the calculation.

Figures 3.21 and 3.22 , shows the Write and Read mode sequence, respectively.

\subsubsection{Interrupt Mode}

There modes give the DTU more freedom and intelligence as of when to contact the DRU. It often happens that the DTU have an important message to send to DRU i.e. alarm, so it shouldn't wait for its turn to be polled. Depending how important this message is it can interrupt the DRU process and communicate this message.

If more than one DTU attempts to interrupt the DRU process, the DRU will handle them according to the priorities which are pre-assigned to them. In our system we 


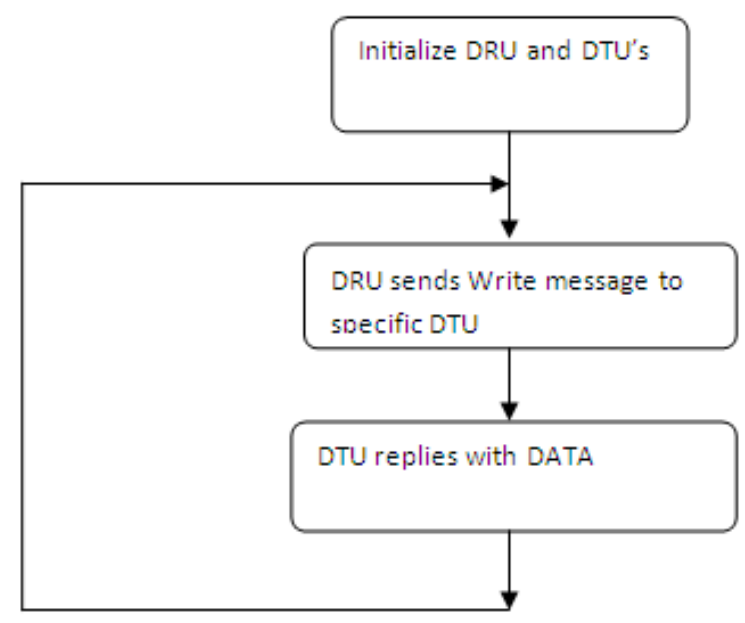

Figure 3.21: Write Mode Sequence

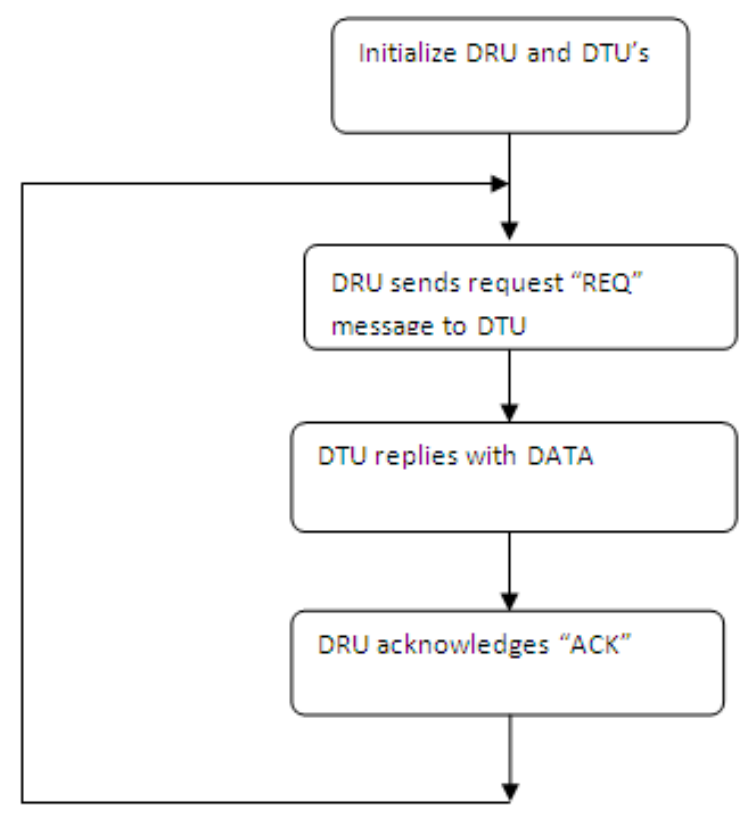

Figure 3.22: Read Mode Sequence 
assume the interrupts coming from fans and sensor have the highest priority. This is mainly due to the fact that fans and sensors functionality directly affects the health and safety of miners, as high pollutants in the mine environment may lead to cases of severe illness and death among miners. The second priority level was assigned to interrupts from amplifiers. Amplifier failure will cause an outage in the underground network and will disrupt not only voice but all other services as well. Third priority level was given to PSU since they are usually robust and has back-ups. Fourth priority level exists but it is reserved for future use.

More on Interrupt mode can be found in the next chapter (Chapter 4: Ventilation on Demand). A the Interrupt mode is the foundation layer for the Ventilation-onDemand. 


\section{Chapter 4}

\section{Ventilation-on-Demand}

\subsection{Introduction}

Ventilation is a very important aspect in mines as it directly affects the health and safety of miners. Accumulation of poisonous gasses and particles in mines can cause serious illness to mines and explosions underground. Trying to cut down their cost in such economy and become more environmentally responsible, mining companies found that ventilation counts for a huge portion of their energy consumption. Ventilation on demand is a hot topic that has been newly researched by mining companies for the last few years.

Ventilation in mines happens by circulating air from above ground to underground. This method keeps fresh air flowing into the mine from above ground and pushing the air underground - which contain gases and dust- out of the mine. The mine ventilation system is made of three main components:

1. Main ventilation system and fans

2. Level ventilation system and control 
3. Auxiliary systems and fans

The main ventilation system and fans will bring the fresh air from above ground to underground. It keeps working $24 / 7$ non-stop to secure mine air needs. Then auxiliary systems and fans take air to serve specific areas in the mines. Each auxiliary system and fans is responsible for a certain area i.e. a mine gallery at a certain level. Switching off this auxiliary system doesn't affect the main ventilation system and fans.

Currently, in all mines the fan's auxiliary systems are always on. Either this mine gallery has miners working in it or not. This results in huge energy waste as miners are not working in all mine galleries at the same time, While energy is very limited and expensive underground.

Our idea here is to switch the fans on/off and increase/decrease their speed according to mine needs. No extra hardware is required since we are going to use the diagnostic system explained earlier in Chapter 3, which makes use of the already existing infrastructure of leaky feeders.

\subsection{Ventilation on Demand Using Diagnostic Sys- tem}

It is the power saving feature that is built on top of interrupts. Ventilation network in mines serve the critical task of maintaining a low concentration of explosives or poisonous gases. It is such important equipment for safety that it will be a huge threat to safe production if it does not meet the needs of mine production for some 
reason [20]. Due to the objective of controlling fluid flow, mine ventilation networks are high-order nonlinear systems [8]. Some models were developed for ventilation networks, however; the idea of ventilation on demand was hardly tackled before.

It is a novel idea that will help cut the operation expenses and attain huge power savings. In most mines the ventilation fans are kept on 24 hours a day in all sections whether miners are working in these sections or not. Ventilation is responsible for about $60 \%$ of power consumed in a mine which drains the limited resource of electricity.

This problem is solved by using the currently available sensors and fans in mines and attaching to each of them a DTU. Sensors and fans will keep track of the pollutant levels in air and temperature as well. Whenever temperature and/or level of toxic gases in this section hits a predefined threshold DTU attached to this sensor and fan will interrupt DRU resulting in the fans being turned on and/or in increasing the fan speed. Then during the normal reading polling cycle if the DRU reads low or normal pollutant level the fans can be turned off and/or their speed decreased.

\subsection{Interrupt}

At any time the DTU can interrupt the process of the DRU to send to it an important message. Messages will have different priority levels depending on their source i.e. amplifier, PSU, fan, and sensor. The interrupt process is shown in Figure 4.1 and is described as follows: 
- DTU sends out interrupt (INT) message containing information about the node,

- DRU pauses current process, and checks type of interrupt also check priority if more than one interrupt,

- DRU replies with (WRITE) message, then goes back and resumes the current process.

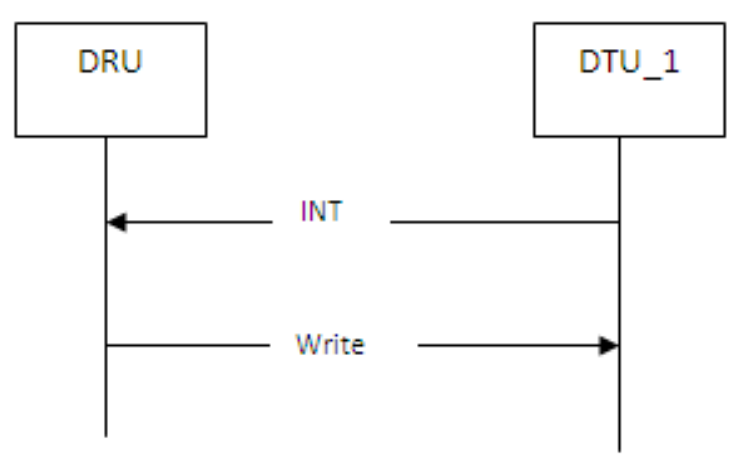

Figure 4.1: Interrupt Message Sequence

If more than one interrupt is received at the same time, the DTU handles the highest priority first while all other priorities are being queued in sequence according to their priority level. Furthermore, in case more than one interrupt is received with the same priority level then the DRU handles the interrupt with lower penalty. Penalty is defined as the time the DRU takes to stop communicating with the current DTU and establish communication with the DTU that issued the interrupt. In our protocol we assumed a correlation between the physical location of the DTU that issued the interrupt and the location of the DTU currently communicating with DRU. For example, if DRU is currently communicating with a DTU (DTU \#1) and two DTU's issue interrupts (DTU \#2 and DTU \#3). If distance between DTU \#2 and DTU \#1 is 300 meters while the distance between DTU \#3 and DTU \#1 is 1200 
meters, then DTU \#3 has higher penalty. Thus, interrupt coming from DTU \#2 would be handled first.

The messages sent and received in the Interrupt mode are as follow:

INT: Interrupt message

Purpose: DTU initiates the interrupt message any time to send any important message to the DRU. Generally, if there is an alarm DTU shouldn't wait for its turn to communicate rather it just interrupts and the DRU handles it.

\begin{tabular}{|c|c|c|c|c|c|c|c|}
\hline $\begin{array}{c}\text { Preamble } \\
\text { bits }\end{array}$ & $\begin{array}{c}\text { Source } \\
\text { Address }\end{array}$ & $\begin{array}{c}\text { Destination } \\
\text { Address }\end{array}$ & Control & Priority & DATA & Reserved & CRC \\
\hline
\end{tabular}

Figure 4.2: Interrupt Message Format

- Preamble: 16 bits for synchronization and indicating the start of the frame.

- Source address: 8 bits indicating the message origin. Here it will be DTU originator address. 8 bits means that the system supports maximum 256 nodes.

- Destination address: 8 bits indicating the receiver address. Here it will be the intended DRU. 8 bits means that the system supports maximum 256 nodes.

- Control: 8 bits

- Bits 0-1: device type: PSU $=00$, Amplifier $=11$, fan and sensor $=10$

- Bits 2-4: Represents the type of the message as shown in Table 3.4. Here, it is a INT message with the value INT=110 
- Bit 5-7: reserved

- Priority: 2 bits indicate the importance of the message, if more than one message sent they are held in sequence and the message with higher priority is handled first.

- Low priority $=00$

- Normal priority "default" here we will set it for PSU $=01$

- Medium priority, we will set it for amplifiers $=10$

- High priority, we will set it for fans and sensors $=11$

- Data: 9 bytes as shown in 4.1

Table 4.1: Data Read During Interrupt Mode-Read Message

\begin{tabular}{|l|l|l|l|}
\hline \multicolumn{4}{|c|}{ Read Cycle } \\
\hline & Amplifier & Battery & Fan and sensor \\
\hline Byte 1 & RF level \#1 & DC voltage & Air quality (sensor) \\
\hline Byte 2 & RF level \#2 & DC current & ON/OFF (fan) \\
\hline Byte 3 & RF level \#3 & AC voltage & fan speed \\
\hline Byte 4 & Amplifier gain \#1 & Temperature & Reserved \\
\hline Byte 5 & Amplifier gain \#2 & Hours of operation & - \\
\hline Byte 6 & Amplifier gain \#3 & Battery voltage & - \\
\hline Byte 7 & Amplifier DC voltage & Battery backup status & - \\
\hline Byte 8 & Amplifier DC current & Reserved & - \\
\hline Byte 9 & Reserved & - & - \\
\hline
\end{tabular}

- Reserved: 8 bits reserved for future use

- CRC: 16 bits check sum. Preamble bits are not included in the calculation

WRITE message

Purpose: For DRU to write data to specific DTU. i.e. change parameters of amplifier, 
PSU, fan, and sensor.

It is the same WRITE message in Figure 3.16, with the below specifications.

- Preamble: 16 bits for synchronization and indicating the start of the frame

- Source address: 8 bits indicating the message origin. Here it will be DRU address. 8 bits means that the system supports maximum 256 nodes.

- Destination address: 8 bits indicating the receiver address. Here it will be the intended DTU. 8 bits means that the system supports maximum 256 nodes.

- Control: 8 bits

- Bits 0-1: device type: PSU $=00$, Amplifier $=11$, fan and sensor $=10$

- Bits 2-4: Represents the type of the message as shown in Table 3.4. Here, it is a WRITE_DATA message with the value WRITE_DATA=110

- Bit 5-7: reserved

- Priority: 2 bits indicate the importance of the message, if more than one message sent they are held in sequence and the message with higher priority is handled first.

- Low priority $=00$

- Normal priority "default" here we will set it for PSU $=01$

- Medium priority, we will set it for amplifiers $=10$

- High priority, we will set it for fans and sensors=11

- Data: 4 bytes as shown in Figure 4.2 
Table 4.2: Data Configured During the Write Cycle-Interrupt Mode

\begin{tabular}{|l|l|l|l|}
\hline \multicolumn{3}{|c|}{ Write Cycle } \\
\hline & Amplifier & Battery & Fan and sensor \\
\hline Byte 1 & Amplifier Gain \#1 & ON/OFF & Fan ON/OFF \\
\hline Byte 2 & Amplifier Gain \#2 & Switch (Primary/ backup) & Increase/ decrease fan speed \\
\hline Byte 3 & Amplifier Gain \#3 & Reserved & Reserved \\
\hline Byte 4 & Reserved & - & - \\
\hline
\end{tabular}

- Reserved: 8 bits reserved for future use

- CRC: 16 bits check sum. Preamble bits are not included in the calculation

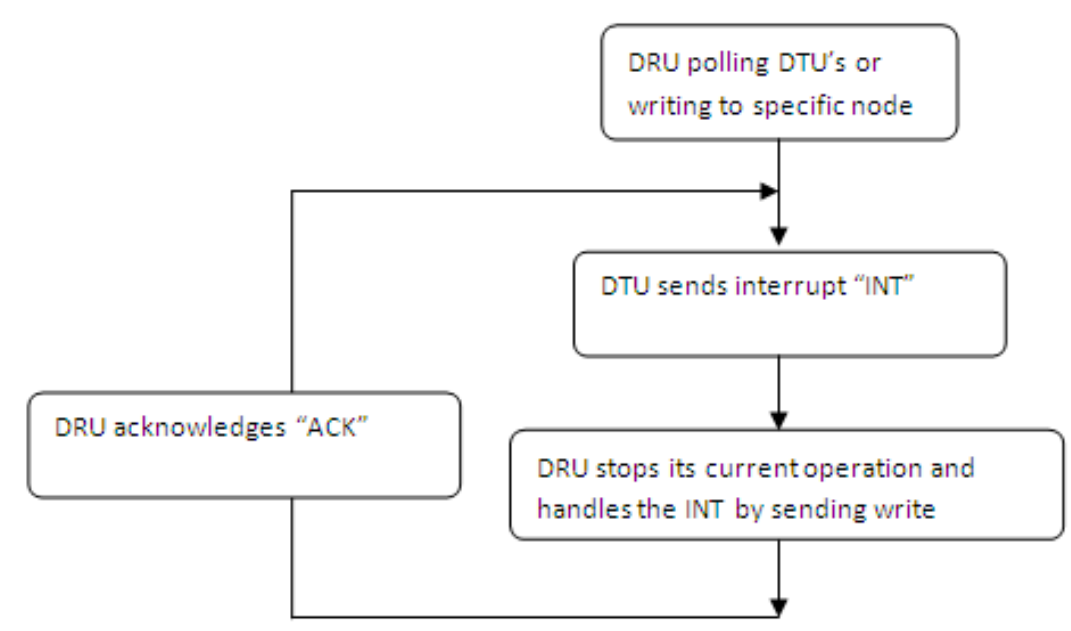

Figure 4.3: Interrupt Mode Sequence 


\section{Chapter 5}

\section{Performance Evaluation}

\subsection{Introduction}

Evaluation for the DS and its communication protocol is presented in this chapter. Here, we use simulations to prove the high performance and efficiency of the system. Under regular mine conditions we calculate SNR, delay, wake up time for each node, and throughput. Also two distinguished types of errors that affect communications in mines and may degrade the performance of the DS are investigated here: Components Tolerance Error (CTE) and Gain Estimation Error (GEE).

For simulation purposes, we consider a typical leaky feeder channel which is modeled as a coaxial cable with mine errors and distortion in addition to white noise. For errors and noise we used pre-measured values from a standard mine gallery.

Propagation delay in leaky feeders depends on capacitance and inductance. These can be obtained using Vector Network Analyzer (VNA) or from the manufacturer

data sheet. In our simulation we assumed a standard 7/8" low loss dielectric leaky 
feeder cable with inductance $\mathrm{L}=0.185 \mu \mathrm{H} / \mathrm{m}$ and capacitance $\mathrm{C}=74 \mathrm{pF} / \mathrm{m}$. This is a standard leaky feeder cable widely used in current systems in mines. Using equation 5.1.1 delay in leaky cable is calculated. It is found that Delay=3.7 $\mathrm{nsec} / \mathrm{m}$.

$$
\text { Delay }=\sqrt{L * C}
$$

System size $(S)$, is defined as the number of active DTU's in the mine. Active DTU's are those attached to amplifiers, PSU, fans, and sensors and are in working condition. Considering the system configuration in Table 5.1, $S N R=-22$.

Table 5.1: System Configuration

\begin{tabular}{|l|l|l|l|}
\hline System Size $(S)$ & Amplifiers & PSU & Fans and sensors \\
\hline 256 & 105 & 63 & 88 \\
\hline
\end{tabular}

In our efforts to prove the ability of the self configuration mode to detect the network layout and to calculate losses in the system we used the following system setup in Figure 5.1.

In Figure 5.1, $n$ represent section number. We experimentally measured actual values and compared them with Matlab simulation output. The simulation results were almost identical to the measured values as shown in Tables 5.2 and 5.3. It was found that the self configuration mode algorithm was able to detect the network layout correctly in terms of number of nodes, distance between nodes, number of splitters, and losses. 


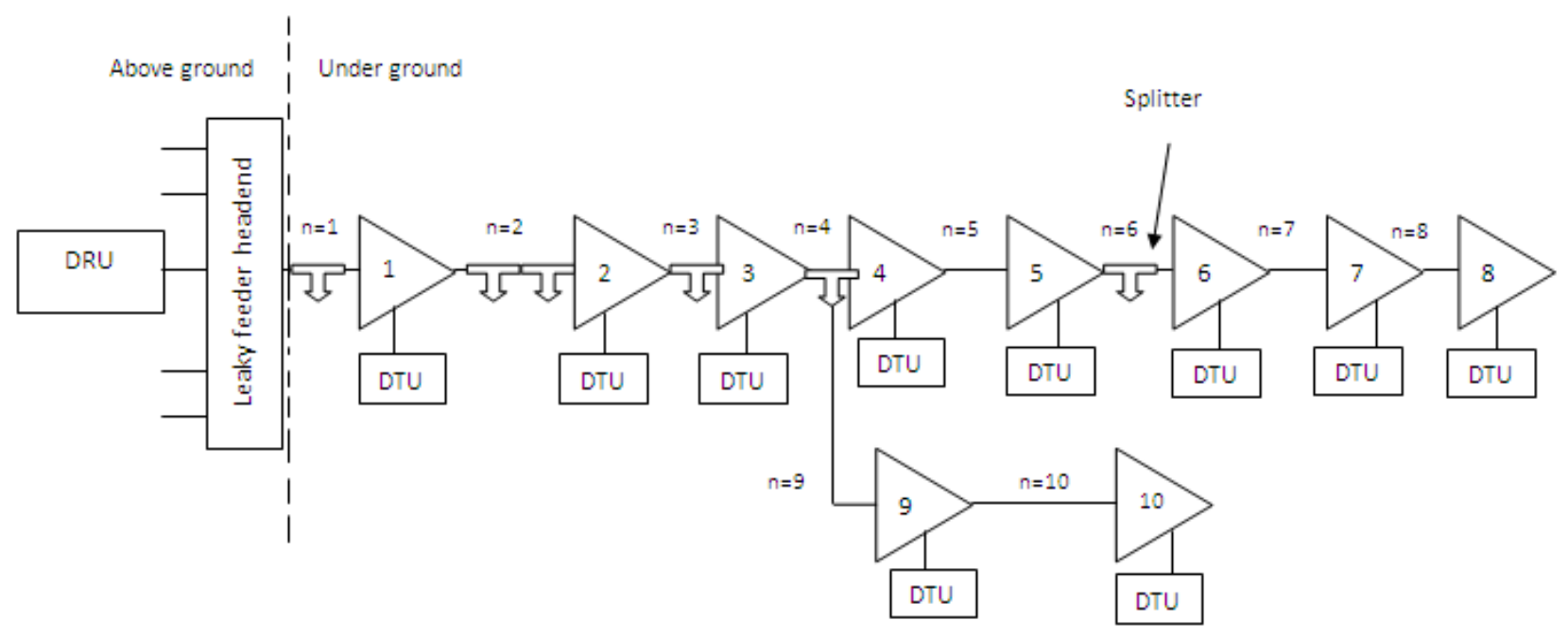

Figure 5.1: Leaky Feeder System Setup

\subsection{Wake up Time}

Using simulations we calculate the percentage of wake up time to the total time of each DTU. This show how long is the DTU in sleep mode compared to wake up mode which gives a good indication of power consumption. Figure 5.2, shows the time each node will stay awake compared to the total cycle time in case of self configuration mode. We notice that when the system size $S$ is around 100 nodes each node will be awake for about $0.9 \%$ of the total cycle time. We also notice that DTU's connected to amplifiers, PSUs, fans, and sensors will exhibit similar behavior due to the nature of self configuration mode that the DRU is sending the same messages of the same length to all nodes in order to discover the network layout.

Wake up time to the total time for the Normal mode- writing cycle case is shown in Figure 5.3. From that Figure it is noticed that the percentage of wake up time for both PSU, and fans and sensors are almost identical but is higher for amplifiers. 
Table 5.2: Measured Values vs. Simulation Results part 1/2

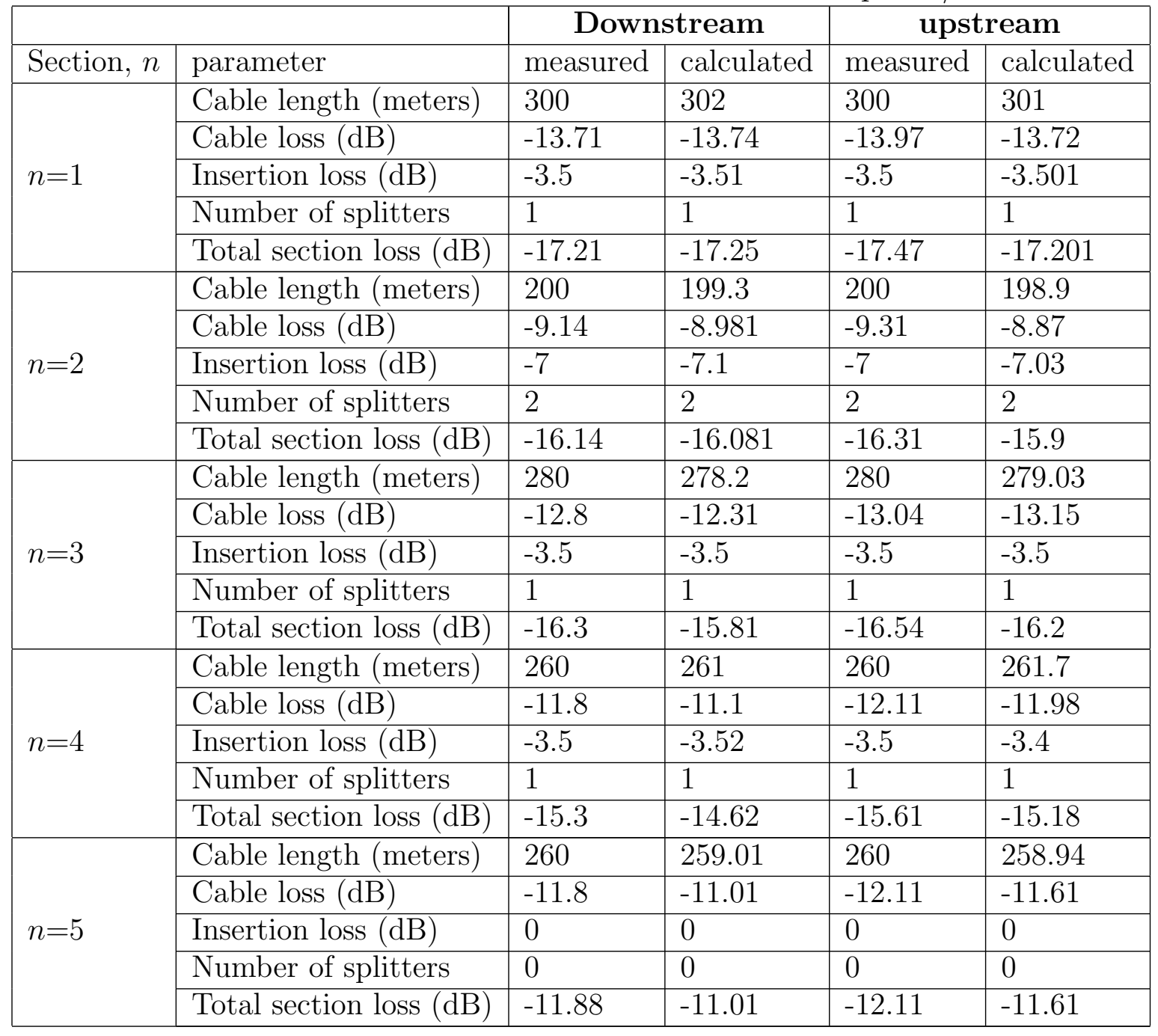


Table 5.3: Measured Values vs. Simulation Results part 2/2

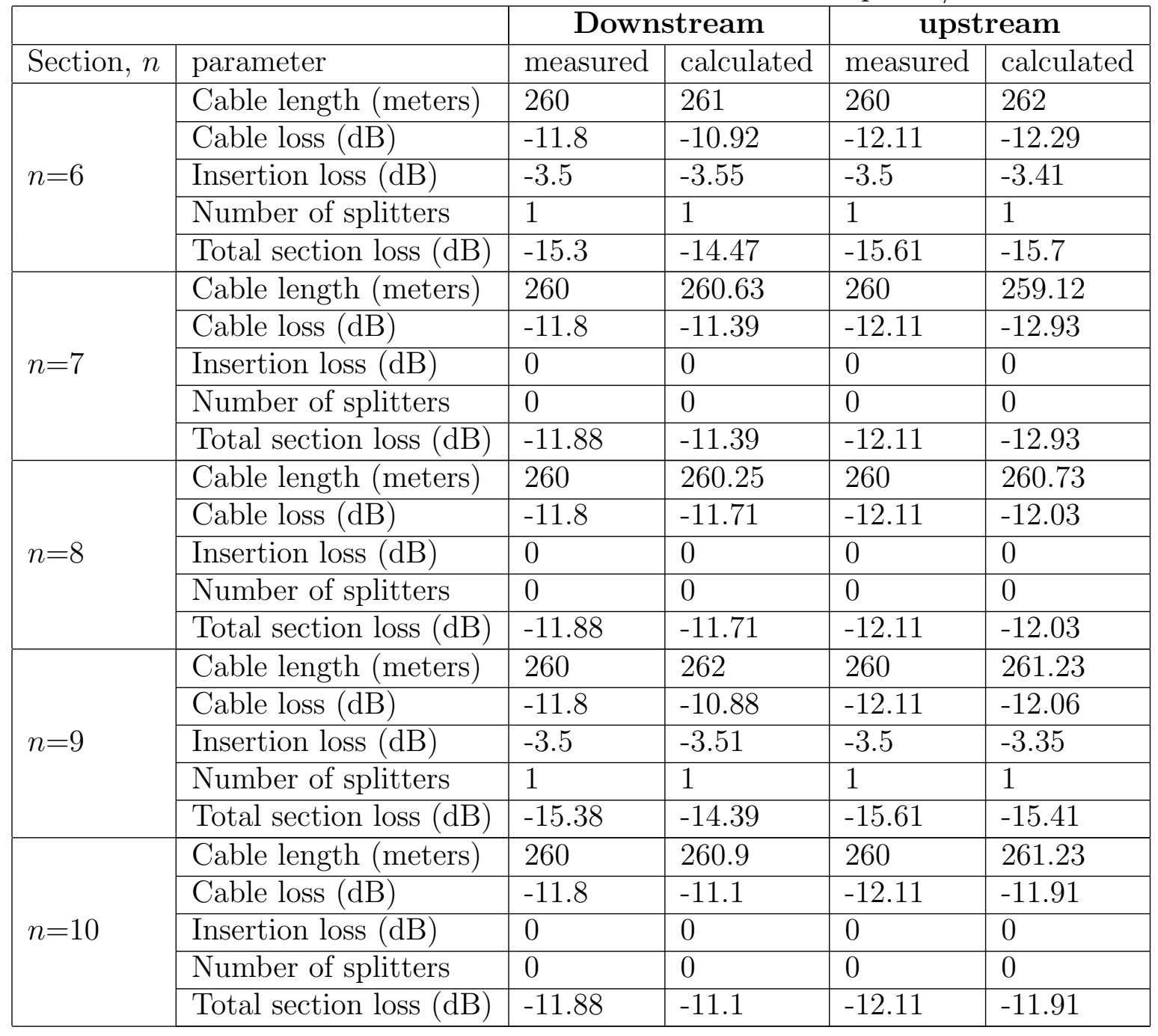




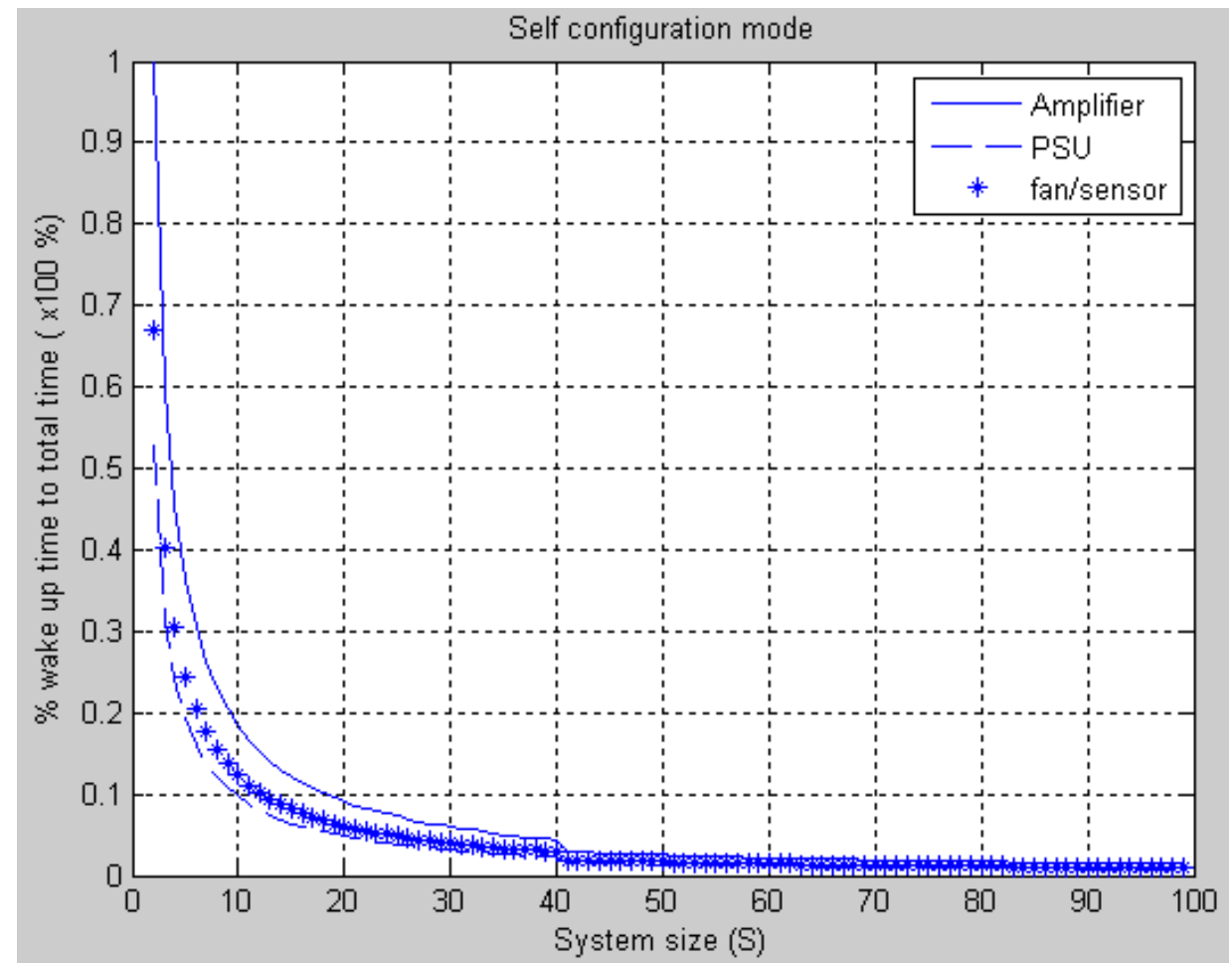

Figure 5.2: Percentage of DTU Wake up Time to Total Time for Different System Sizes- Self Configuration Mode 


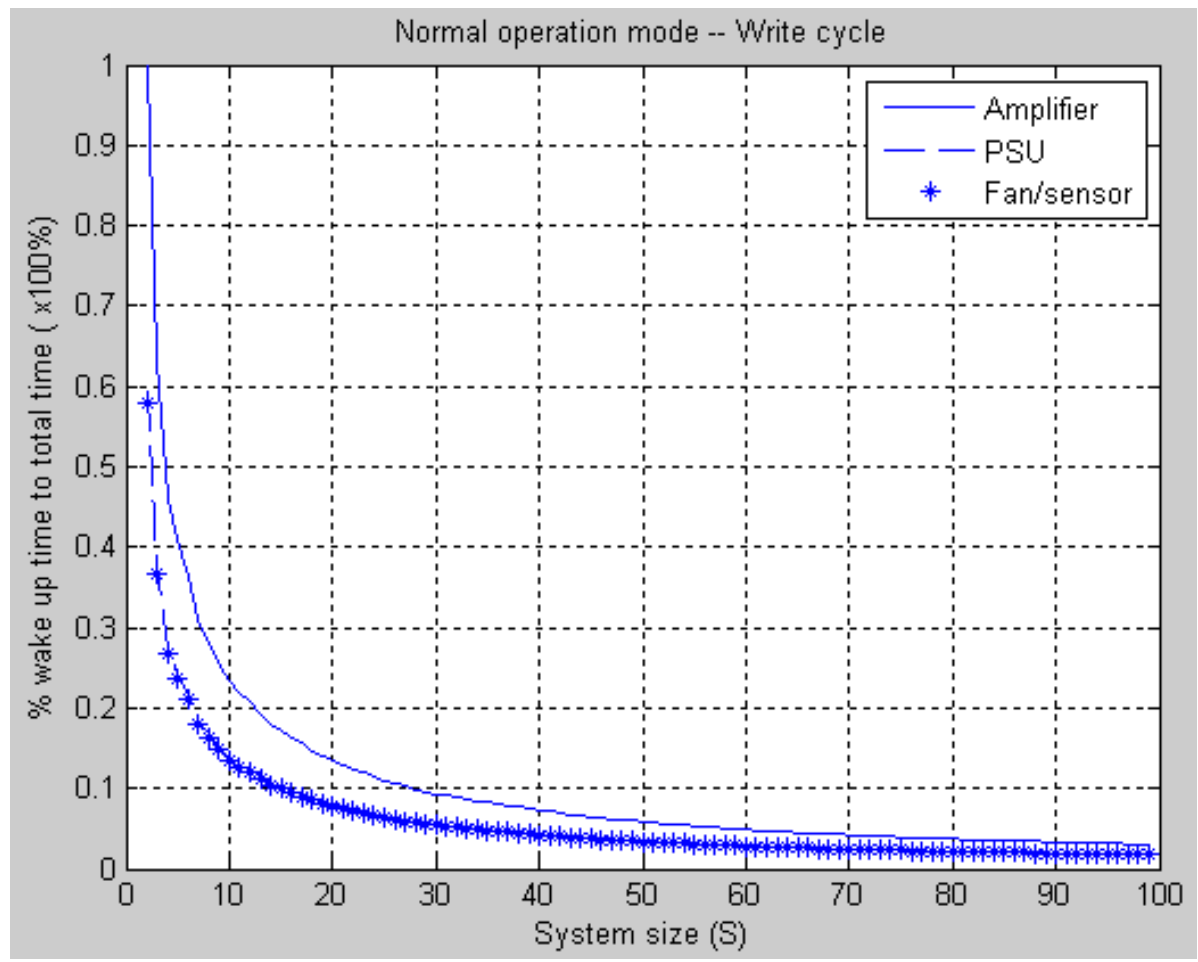

Figure 5.3: Percentage of DTU Wake up Time to Total Time for Different System Sizes- Normal Mode- Write Cycle

The difference in percentages is due to amplifier messages being longer than the other messages sent to PSU or fans and sensors.

Figure 5.4, shows the time each node will stay awake compared to the total time of a complete reading cycle, when the DRU polls all DTUs. Again we notice that the amplifiers wake up time is longer than other nodes due to the longer messages used in case of amplifiers compared to PSU or fans and sensors.

For interrupt mode we show the penalty paid compared to the system size, $S$. In Figure 5.5, the $S=256$ and 82 random interrupts are coming from random nodes at different times. As described earlier, penalty can be defined as extra time to handle 


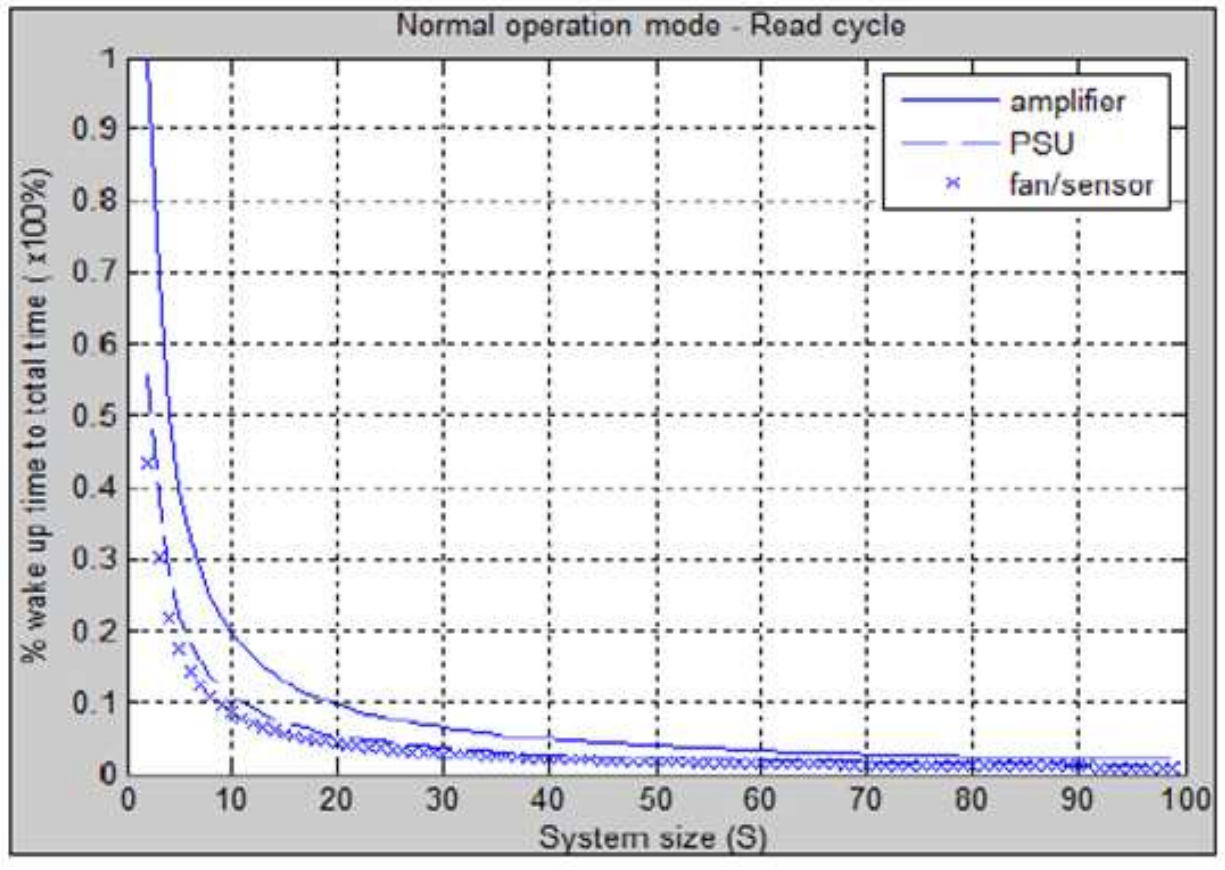

Figure 5.4: Percentage of DTU Wake up Time to Total Time for Different System Sizes- Normal Mode- Read Cycle 


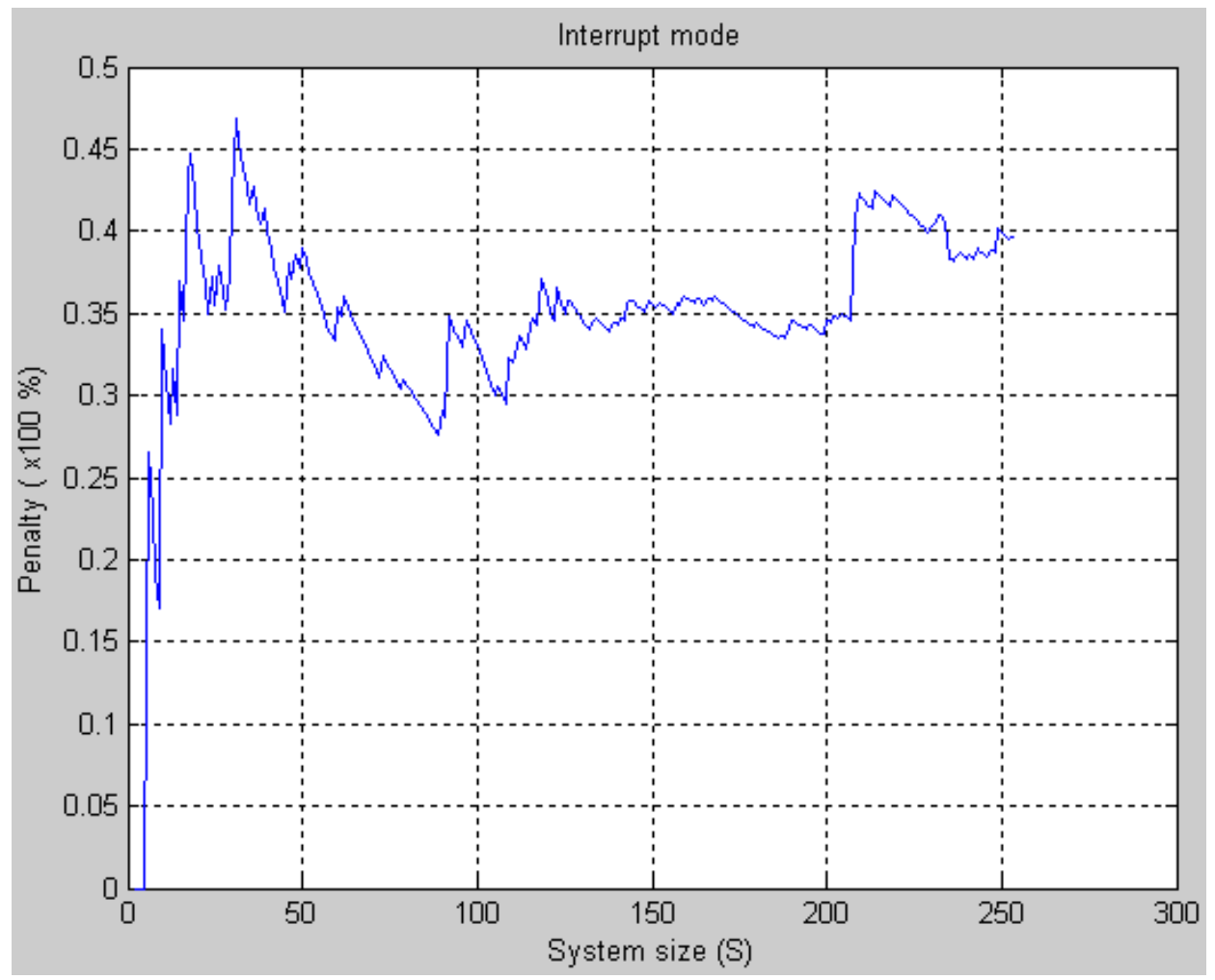

Figure 5.5: Extra Processing Time for Different System Sizes- Interrupt Mode

the interrupt. From Figure 5.5, maximum penalty is $47 \%$ extra processing time. Penalty $=$ Total time- Cycle execution time with no interrupts

\subsection{End-to-End Delay and Throughput}

Another important parameter is end-to-end delay which is extremely important as it indicates the overall response of the system. The operator does not want to wait so long to collect some critical information. The end-to-end delay was calculated for one hour period where we assumed that self configuration mode ran at the beginning then the writing mode took place for 10 minutes where we kept writing to nodes in a random manner, and for the rest of the hour DRU was polling DTU's in sequence to 


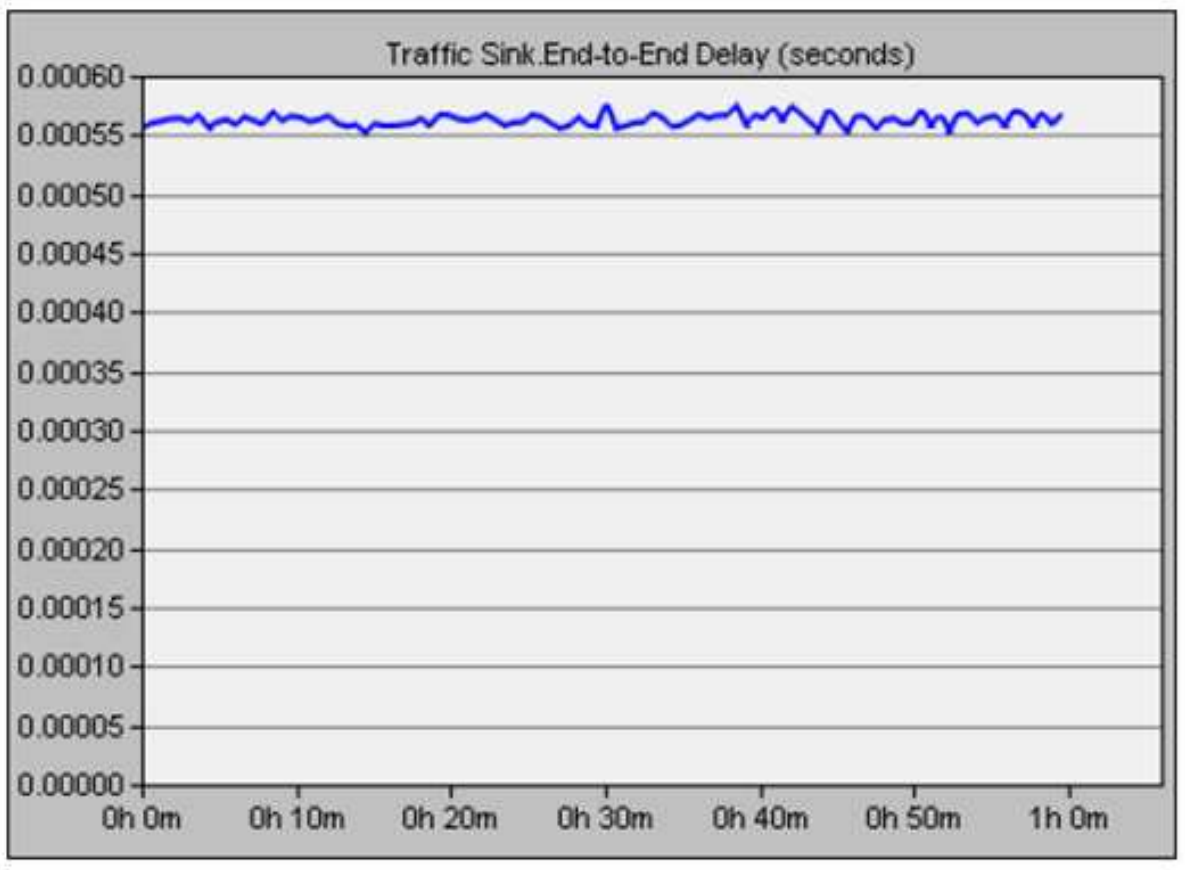

Figure 5.6: Average End-to-End Delay in the Network Over One Hour

read their data. Figure 5.6 shows that the average end-to-end delay is in the range of tens of milliseconds which is acceptable for underground mine applications.

Under the same circumstances we calculated the average throughput in the system and the results are shown in Figure 5.7. The average throughput was found to be in the range of few hundred bits/sec which means that the bandwidth allocated is not fully utilized and the system still has room for more messages. 


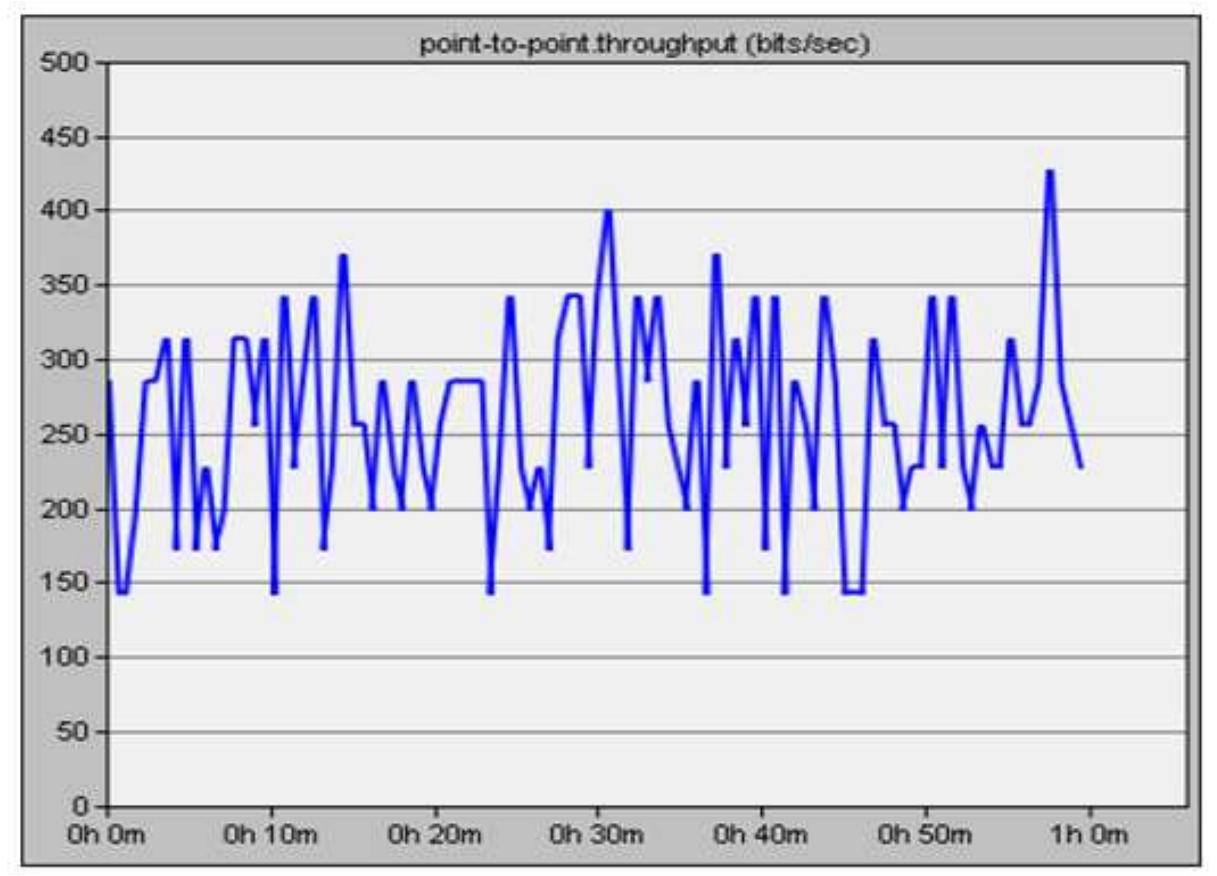

Figure 5.7: Average Throughput in the Network over an Hour

\subsection{Component Tolerance Error (CTE)}

Components Tolerances Error (CTE) is when the accumulations of components tolerances in electronics circuits cause part or all of the circuit to operate outside its nominal operating range [7]. The errors related to tolerance of circuit components can be reduced if the circuits are properly calibrated during manufacturing.

However, because of the extreme harsh conditions in mines and tunnels, the components might change after system installation especially because of temperature. This can affect the functionality of the DS and its communication protocol. CTE mainly affects the self configuration mode where pilot signals are used. Ignoring CTE may result in imprecise pilot signals being sent from DRU to DTUs. Therefore, the 
operator will get wrong information about the network layout in terms of losses, distance between nodes, number of nodes and splitters.

CTE factor $\left(E_{C T E}\right)$ is defined as the percentage of errors in the gains of the amplifiers caused by CTE.

$$
E G_{m}=G_{m} \cdot\left(1+E_{C T E}\right)
$$

$E G_{m}$ : Errored gain of the amplifier

$G_{m}$ : Nominal error-free gain of the amplifier

$E_{C T E}$ : Component tolerance error factor

CTE in mines is usually around $2.5 \%$ which is high. In order to solve this problem we added an additional RF detector at the output stage of the downstream nodes. The output of the RF detectors is fed into the DTU microprocessor for comparison with the reference pilot signal. Difference between reference pilot signal and the one detected by the extra RF detector is calculated and used to correct the amplifier gain.

$$
\begin{gathered}
C F_{m}=P L_{\text {Reference, } m}-P L_{R F \text { detector }, m} \\
C G_{m}=E G_{m}+C F_{m}
\end{gathered}
$$

$C F_{m}$ : Correction factor

$P L_{\text {Reference, } m}$ : Reference pilot level at frequency, $m$

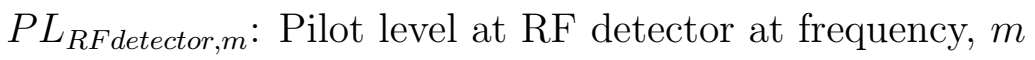

$C G_{m}$ : Corrected gain of the amplifier at frequency, $m$

By adding Correction Factor to the amplifier's errors gain the correct gain can be determined. Figure 5.8 shows in blue trace the amplifier output with uniformly 


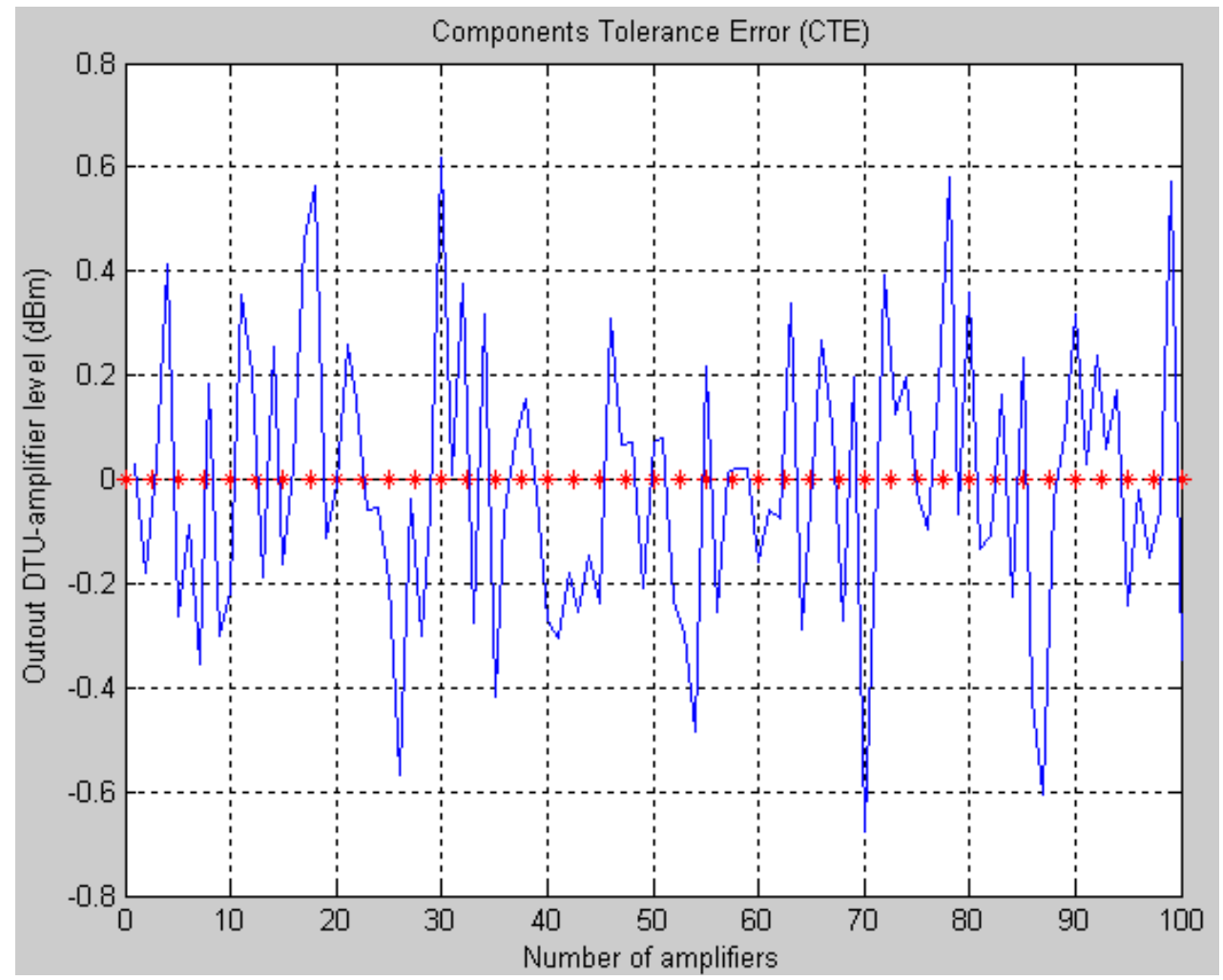

Figure 5.8: CTE Simulation Analysis

distributed random $\mathrm{CTE} \pm 2.5 \%$ i.e. $5 \%$ versus corrected gain amplifier output with red trace, assuming self configuration mode is running where the pilot signal sent is $0 \mathrm{dBm}$. From Figure 5.8 it is clear that this technique provides $100 \%$ correction for pilot amplifier output where the corrected output is self-healed and gives $0 \mathrm{dBm}$ for all amplifiers while the output in case of no CTE correction keeps varying between \pm $0.5 \mathrm{dBm}$. 


\subsection{Gain Estimation Error (GEE)}

The gain of any amplifier is usually set to compensate the losses in its section. However, sometimes because of faulty cables or bad installation these losses increase. Thus, error may occur when estimating the gain which is not expected to be significant, usually about $5 \%$ in typical mine[7].

But for big systems with large number of amplifiers these errors add up and change the behavior of the amplifier. This change is either the output of the amplifier exceeding the rated maximum output power thus saturating the amplifiers or the amplifier output is less than the receiver sensitivity thus no signal is detected.

Here, we show that our diagnostic system is GEE-proof where the maximum output of amplifiers is $-10 \mathrm{dBm}$ and the minimum output is $-80 \mathrm{dBm}$, assuming a voice signal -24 dBm and 5\% GEE over 100 amplifiers. Figure 5.8, shows acceptable system operating conditions where the amplifier output keeps oscillating within the safe range and never exceeds the maximum $-10 \mathrm{dBm}$ or hits the minimum $-80 \mathrm{dBm}$. Amplifier output is shown in blue trace while voice signal power is shown in red trace. 


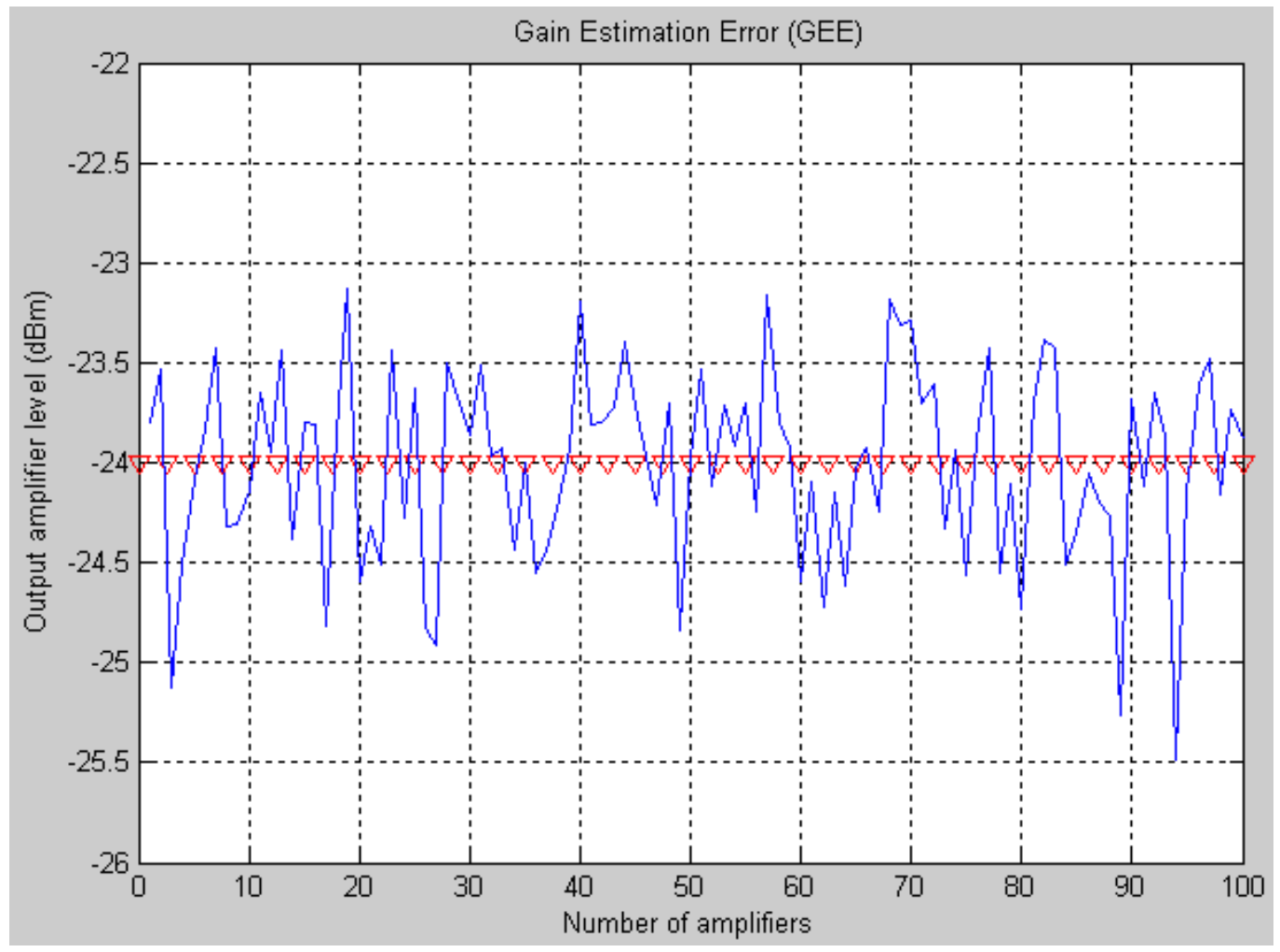

Figure 5.9: GEE Simulation Analysis 


\section{Chapter 6}

\section{Conclusion and Future Work}

\subsection{Conclusion}

Theoretical analysis was conducted to evaluate and study a complete and integrated monitoring system for underground leaky feeder communication networks. It was concluded that by introducing our diagnostic system along with the communication protocol we developed, we get a novel advanced mine monitoring solution that uses the existing infrastructure to monitor itself.

This was made possible by adding master node DRU at the leaky feeder headend in the control room above ground and few DTU nodes that are connected to the amplifiers, PSU, fans, and sensors. This diagnostic system uses an intelligent communication protocol. It is intelligent not only because of allowing DTUs to read all information of attached nodes and send it to DRU upon request, but also because of giving DRU the flexibility to program any node remotely through the DTU attached to it. 
This diagnostic system provides ventilation on demand through a system of interrupts sent from the DTU to DRU which will provide huge power savings to decrease the overall cost of running the mine.

The analyses of the system behavior have demonstrated its power efficiency, acceptable end-to-end delay, SNR, and throughput. From the throughput we also noticed that the bandwidth is not fully utilized which gives room for more improvement in our communication protocol later on. Performance analyses were done using Matlab, and OPNET and based upon real data collected from a typical mine gallery.

Error analyses for the developed communication protocol considered two significant types of errors that affect operation in mines.

CTE is the first type of error analyzed. It is an error in calculating the gain and slopes of the amplifiers due to tolerance of the amplifier's components [7]. It was concluded that error correction $100 \%$ is possible by adding an extra RF detector at the output stages of the amplifiers.

The second type of error is the GEE, it is the error in estimating the gain for upstream amplifiers band. From the simulation analyses in chapter 4, it was concluded that our communication protocol can ensure reliable operation where the amplifiers in the cascaded system will be operating in acceptable dynamic ranges of operation. 


\subsection{Future Work}

The topic of mine monitoring is so rich in terms of research opportunities and funds available; there is still so much to be done.

\section{Hybrid Mine Monitoring Solution:}

To enhance the environmental-factor monitoring capability a hybrid system should be considered. The hybrid system maybe crucial due to the fact that the underground mine can be divided into two major areas:

1. open area where the extraction activities happen.

2. tunnel areas, which includes passageways and shafts.

The proposed configuration is made of the current leaky feeder as the wired domain and WSN as the wireless domain. The WSN will enhance the coverage and reach out for dead spots that cannot be covered using the wired system only.

The WSN can be integrated to the Leaky Feeder System via Gate Way sensor nodes. In this scheme, clusters of nodes can be integrated into a large scale system via Leaky Cable. In each cluster of nodes, the WSNs employ multi-hop routing to implement data gathering. Each sensor node plays the role of data collector as well as message forwarder in the network. The Gate Way nodes at each cluster will then relay the information into the Leaky Cable network via the CMTS modems. Once on the IP network of the Leaky Cable, the data can be transported a long distance to a central processing facility, where data can be monitored, analyzed, and might be 
processed further for automation actions.

\section{Prototyping:}

It will be advantageous for the developed system to be practically translated into prototype. This will help ensuring the practicality of developing any further systems based on our diagnostic system and communication protocol.

\section{Redundancy:}

Our diagnostic system depends mainly on the DRU located in the control to handle almost all requests. So it will be beneficial to study adding another DRU, a backuplike DRU, to serve as a redundant node in case of DRU failure.

\section{Failure Analyses:}

Failure analyses can further research possible scenarios of failures that can affect the operations of the diagnostic system and the communication protocol. For example DRU hardware failure can be considered. In that case we may want to research the idea of adding redundant DRU in the control room. The communication protocol must be further developed to react in such cases.

\section{Ventilation-on-Demand using RF tagging:}

Most mining companies have an RFID system where they add RF tags to miner's belts' or helmets' and also to all major equipments in mine. This allows tracking of miners and knowing their location exactly. It is worth trying to enhance the Ventilation on Demand system by combining it with the RFID tagging, for instance; 
using RF tags we can determine real time where miners are working so ventilation fans turns on automatically. 


\section{Bibliography}

[1] Robert S. Chapman and Roy S. Nutter, "System Operational Evaluation of Mine Monitoring Systems", IEEE Transactions on Industry Applications IA-21 (1985), no. 5, $1112-1116$.

[2] Robert S. Chapman, Roy S. Nutter, and William R. Voltz, "Test Criteria and Methodology for Evaluation of Coal Mine Monitoring and Control Systems", IEEE Transactions on Industry Applications IA-21 (1985), no. 5, 1117 -1120.

[3] Benessa Defend, Kevin Fu, and Ari Juels, "Cryptanalysis of Two Lightweight RFID Authentication Schemes", Fifth Annual IEEE International Conference on Pervasive Computing and Communications Workshops, 200\%. PerCom Workshops '07, march 2007, pp. $211-216$.

[4] G.Y. Delisle, "Propagation Charactristics for Modern Wireless System Networks in Underground Mine Galleries", International Institute of Telecommunications, Montreal, 2005 First International Workshop on Wireless Communications in Underground and Confined Area (June 6-7, 2005), Val d'Or, QC, Canada.

[5] P. Delogne, "EM propagation in tunnels", IEEE Transactions on Antennas and Propagation 39 (1991), no. 3, $401-406$. 
[6] T.H. Dubaniewicz, J.E. Chilton, and Jr. Dobroski, H., "Fiber optics for atmospheric mine monitoring", IEEE Transactions on Industry Applications 29 (1993), no. 4, $749-754$.

[7] W. Farjow and Fernando X., Leaky feeder system optimization in underground mines and tunnels, MASc Thesis Ryerson University 2009.

[8] Yunan Hu, O.I. Koroleva, and M. Krstic, "Control design for mine ventilation network systems", Proceedings of the 41st IEEE Conference on Decision and Control, 2002, vol. 1, December 2002, pp. 543 - 548 vol.1.

[9] Mining What it means to Canada, Ottawa: The Mining Association of Canada (1988).

[10] Tieyan Li and Robert Deng, "Vulnerability Analysis of EMAP-An Efficient RFID Mutual Authentication Protocol", The Second International Conference on Availability, Reliability and Security, 200\%. ARES 200\%,, april 2007, pp. 238 -245 .

[11] D.J.R. Martin, "Leaky-feeder radio communication: A historical review", Vehicular Technology Conference, 1984. 34th IEEE (1984), 25 -30.

[12] M.F. Mubarak, J.-l.A. Manan, and S. Yahya, "Mutual Attestation Using TPM for Trusted RFID Protocol", Second International Conference on Network Applications Protocols and Services (NETAPPS), 2010, sept. 2010, pp. $153-158$.

[13] T. Novak, D. P. Snyder, and J. L. Kohler, "Postaccident Mine Communications and Tracking Systems", IEEE Transactions on Industry Applications 46 (2010), no. $2,712-719$.

[14] T. Novak, D.P. Snyder, and J.L. Kohler, "Post-Accident Mine Communications and Tracking Systems", Industry Applications Society Annual Meeting, 2009. IAS 2009. IEEE (2009), $1-8$. 
[15] Jr. Nutter, R.S. and M.D. Aldridge, "Status of mine monitoring and communications", IEEE Transactions on Industry Applications 24 (1988), no. 5, 820 $-826$.

[16] B. of Mines Staff, Underground mine communications, control and monitoring, U. S. Department of the Interior, Bureau of Mines, Information Circular $\mathbf{I}$ 28.27:8955 (1985).

[17] K. Osaka, Shuang Chang, T. Takagi, K. Yamazaki, and O. Takahashi, "A Secure RFID Protocol based on Insubvertible Encryption Using Guardian Proxy", Third International Conference on Availability, Reliability and Security, 2008. ARES 08., march 2008, pp. $733-740$.

[18] W. H. Schiffbauer and J. F. Brune, "Underground coal mine communications for emergencies and everyday operation", Symposium on the Capabilities and Availability of Wireless Communication and Tracking Systems for Underground Coal Mines (Feb. 13, 2006).

[19] Jr. Sottile, J. and L.E. Holloway, " An overview of fault monitoring and diagnosis in mining equipment", IEEE Transactions on Industry Applications 30 (1994), no. $5,1326-1332$.

[20] Qingdong Wang, Deyong Shang, Zhiyong Yang, and Changjun Zhu, "Design of coal mine main fan performance optimization", Computational Intelligence and Industrial Applications, 2009. PACIIA 2009. Asia-Pacific Conference on, vol. 2, Novomber 2009, pp. $58-60$.

[21] Craig A. Winkelmann, Andrew M. Kissel, and Roy S. Nutter, "Microprocessor Self-Testing for Mine Monitor Systems", IEEE Transactions on Industry Applications IA-21 (1985), no. 1, $158-161$. 
[22] S. Yarkan, S. Guzelgoz, H. Arslan, and R.R. Murphy, "Underground Mine Communications: A Survey", IEEE Communications Surveys Tutorials 11 (2009), no. $3,125-142$. 


\section{Appendix}

\section{Publications}

1. Daoud, M.; Maheeka, U.; Alam, T.; Fernando, X.; , "Modulator bias optimization of radio over fiber links considering noise figure and RF gain," Communications (QBSC), 2010 25th Biennial Symposium on , vol., no., pp.234-237, 12-14 May 2010.

2. Daoud, M.; Fernando, X.; , "On the communication requirements for the smart grid," Scientific Research Journal, Energy and Power Engineering, vol. 3, no. 1, pp.53-60, 25 February 2011.

3. Daoud, M.; Farjow, W.; Fernando, X.; , "A Novel Diagnostic System for Adding Reliability to Communication Networks in Underground Mines," accepted for the IEEE Canadian Conference on Electrical and Computer Engineering 2011 (CCECE 2011).

4. Daoud, M.; Farjow, W.; Fernando, X.; , "Advanced diagnostic system with ventilation on demand for underground mines," accepted for IEEE Sarnoff symposium 2011.

5. Daoud, M.; Fernando, X.; "On the Communications Requirements for the Smart Grid," accepted for the IEEE Canadian Review-Spring 2011.

6. Daoud, M.; Farjow, W.; Fernando, X.; , "Advanced mine monitoring system with ventilation on demand for underground mines and tunnels," accepted for the IEEE Industry Application Society (IAS) annual meeting 2011. 